\title{
Modeling seafloor geoacoustic interaction with a numerical scattering chamber
}

\author{
Ralph A. Stephen and Stephen A. Swift \\ Woods Hole Oceanographic Institution, Woods Hole, Massachusetts 02543
}

(Received 9 July 1993; accepted for publication 30 March 1994)

\begin{abstract}
A numerical scattering chamber (NSC) has been developed to compute backscatter functions for geologically realistic seafloor models. In the NSC, solutions are computed to the elastic (or anelastic) wave equation by the finite-difference method. This has the following advantages: (a) It includes all rigidity effects in the bottom including body and interface waves. (b) It can be applied to pulse beams at low grazing angles. (c) Both forward scatter and backscatter are included. (d) Multiple interactions between scatterers are included. (e) Arbitrary, range-dependent topography and volume heterogeneity can be treated simultaneously. (f) Problems are scaled to wavelengths and periods so that the results are applicable to a wide range of frequencies. (g) The method considers scattering from structures with length scales on the order of acoustic wavelengths. The process is discussed for two examples: a single facet on a flat, homogeneous seafloor and a canonically rough, homogeneous seafloor. Representing the backscattered field by a single, angle-dependent coefficient is an oversimplification. In a strong scattering environment, time spread of the field is a significant issue and an angle-dependent separation of the wave field may not be valid.
\end{abstract}

PACS numbers: 43.30.Bp, 43.30.Ma, 43.30.Gv, 43.30.Hw

\section{INTRODUCTION}

Low-frequency sound $(50-1000 \mathrm{~Hz})$ can propagate for hundreds of kilometers in the upper ocean before interacting with the seafloor at continental margins or sea mounts (Fig. 1). Observations of basin reverberation indicate that strong backscattered signals from the seafloor can occur for these long-range paths (Preston and Berkson, 1990). Because of the steepness of the corresponding eigenrays, the interaction with the bottom occurs at very low grazing angles, often less than $20^{\circ}$. The physical mechanisms responsible for direct backscatter at these very low grazing angles are poorly understood. This paper outlines a numerical technique capable of predicting the low-frequency acoustic wave field scattered from geologically realistic models of the seafloor. This capability permits the identification of the physical mechanisms that dominate the long-range reverberation from the seafloor and it permits the characterization of the variations in bottom topography and sub-bottom properties that control the scattering of low-frequency acoustic waves. In addition to basin reverberation, the technique can be applied to other scattering problems, such as shallow-water, high-frequency scattering from sediments (Jackson and Briggs, 1992), estimation of seabed properties from multibeam sonar systems (Matsumoto et al., 1993), and scattering from buried objects (Lim et al., 1993).

A theoretical or numerical approach to the strong backscatter problem should address the following issues.

(a) The approach should be able to handle scattering from heterogeneities with scalelengths on the order of acoustic wavelengths. Strong, long-range backscatter is observed in the frequency range $50-1000 \mathrm{~Hz}$ and there is a great deal of structure on the seafloor that has scalelengths comparable to acoustic wavelengths at these frequencies. (b) Both interface and volume scattering should be treated in the same formulation so that a direct comparison can be made between the relative effects of each.

(c) The method should consider energy incident on the bottom at low grazing angles $\left(<20^{\circ}\right)$ since these are the eigenray angles that dominate long-range propagation.

(d) The method should include shear-wave effects including both body and interface waves.

(e) The method should treat two-way effects including both forward scatter and backscatter.

(f) Multiple interactions between scatterers should also be allowed.

(g) The energy lost or gained from interface waves, at the seafloor and the sediment/basement contact, can be significant for a given scattering problem and should be considered in the scattering model.

The finite-difference method applied to the solution of the two-way elastic (or anelastic) wave equation lends itself well to this problem. By introducing a "pulse beam" in a "numerical scattering chamber" (Stephen, 1991; Stephen, 1993, Fig. 2; Stephen and Dougherty, 1993), all of the above criteria can be met. Also by applying a beamforming algorithm to the output of the NSC we can compute curves of scattering coefficient versus grazing angle for incident beams at a given angle. This allows us to compare quantitatively the scattering responses for a broad range of seafloor structures.

\section{BACKGROUND MATERIAL}

\section{A. The finite-difference method for seismo-acoustic problems}

The historical development of the finite-difference method applied to seismo-acoustic problems goes back over 


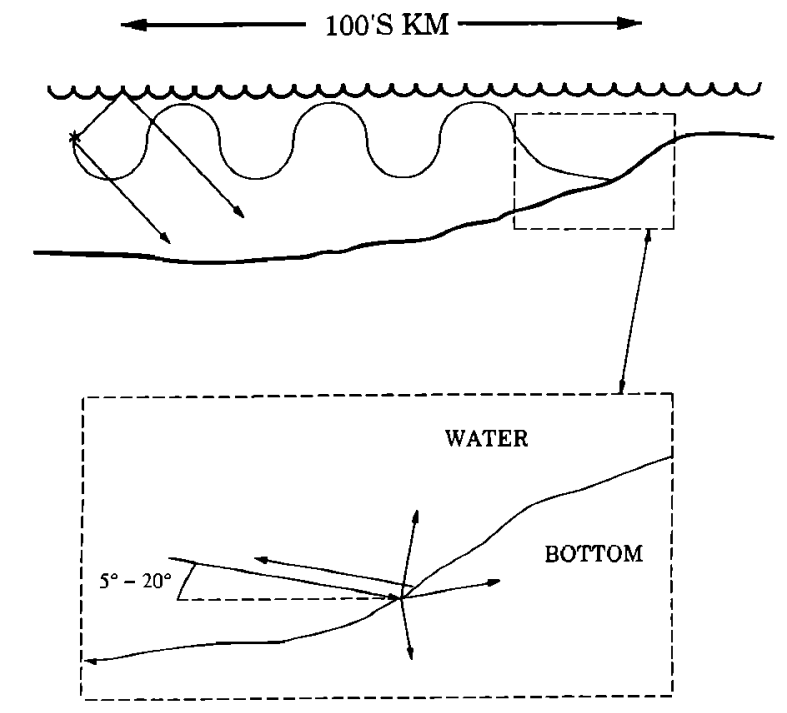

FIG. 1. Long-range sound propagation can be represented by eigenrays trapped in the surface layers of the ocean. Strong backscatter occurs when this energy hits the bottom at a continental margin or seamount. The sound hits the seafloor at grazing angles less than about $20^{\circ}$ and significant energy is returned directly back along the incident path. The numerical scattering chamber was developed to study the physical mechanisms responsible for long-range, low-angle backscatter from the seafloor. This figure is from Stephen and Dougherty (1993).

25 years (e.g., Alford et al., 1974; Alterman and Karal, 1968; Boore, 1970; Kelly et al., 1976) and the field is still an active area of research (e.g., Fricke, 1993; Leslie and Randall, 1992; Yoon and McMechan, 1992). A collection of some of the early papers has been compiled by Kelly and Marfurt (1990). Depending on the application, various investigators apply finite differences to different two-way wave equations and Stephen (1988) gives a summary of these up to 1988. (We distinguish here between the "wave equation" in the time domain and the "Helmholtz equation" in the frequency domain.) Many formulations prior to 1986 had stability problems at sharp, rough fluid-solid boundaries but the offsetgrid methods (Madariaga, 1976; Virieux, 1986) show considerable improvement. In addition to straight second-order finite differences, there are related but alternative approaches such as higher-order methods (e.g., Dablain, 1986; Levander, 1988), the pseudospectral method (e.g., Gazdag, 1981; Kosloff and Baysal, 1982), and the finite-element method (e.g., Teng and Kuo, 1988). The method of finite differences has also been applied to one-way or parabolic-wave equations (e.g., Claerbout, 1970; Gazdag, 1981; Lee et al., 1981). For continuous-wave problems in purely acoustic media, the Helmholtz equation can be conveniently solved in the frequency domain using finite-element methods (e.g., Gan and Ludwig, 1993; Murphy and Chin-Bing, 1989).

One challenging aspect of computing two-way wave equation solutions on a finite spatial domain is the implementation of the Sommerfeld radiation condition (that is, absorbing boundaries). A significant development in this area was the application of the parabolic approximation at the boundary (Clayton and Engquist, 1977; Engquist and Majda, 1977), but the stability of the early formulations was sensitive to Poisson's ratio (Emerman and Stephen, 1983). Hig- don $(1986,1991)$ extended the parabolic approximation approach in a stable scheme and Peng and Toksöz (1994) present a method for determining optimal coefficients in these schemes. Another popular absorbing boundary approach is to add damping directly to the wave equation in a region surrounding the domain (e.g., Cerjan et al., 1985; Levander, 1985).

A major disadvantage of the finite-difference method is the computational effort required to obtain accurate solutions. Depending on the problem, between 10 and 60 grid points per wavelength are required for acceptable accuracy (Alford et al., 1974; Dougherty and Stephen, 1991). Typical grid sizes for elastic solutions cover a propagation region of about $100 \times 100$ wavelengths for two-dimensional problems and of about $30 \times 30 \times 30$ wavelengths for three-dimensional problems. To double the frequency for the same problem dimensions in space and time requires eight times the computational effort for a two-dimensional problem. The inclusion of anelastic effects can also increase the computational effort considerably (e.g., Day and Minster, 1984; Emmerich and Korn, 1987).

A second disadvantage of the finite-difference method, which it shares with many numerical approaches, is the difficulty of confirming accuracy for complex problems. The technique has the potential to obtain solutions to problems for which no analytical solutions exist, but the accuracy of the solutions is not guaranteed. So for a given class of problems, results are computed by a number of numerical approaches and if the results agree the solution is assumed to be correct (e.g., Priolo et al., 1994). Stephen (1983) compared finite-difference solutions to reflectivity (discrete wave number) solutions for range-independent seafloor problems with velocity gradients and showed that even for these simple models various finite-difference formulations could give erroneous results. Stephen (1990) demonstrated excellent agreement between finite-difference solutions and analytical and normal-mode solutions for various rangedependent, fluid-wedge models. Similar benchmark studies should be carried out for rough-interface and volumescattering models.

\section{B. Scattering at penetrable, rough, elastic seafloors}

Although the numerical scattering chamber can be applied to a broad range of two-dimensional scattering problems, the examples in this paper deal with scattering of energy incident at small grazing angles from randomly rough, penetrable, elastic seafloors. Alternative methods for solving this problem include perturbation methods (Chuang and Johnson, 1982; Dacol and Berman, 1988; Kennett, 1972; Kuperman and Schmidt, 1989), Rayleigh methods (Berman and Perkins, 1990), Kirchhoff methods (Ogilvy, 1987), boundary-integral methods (Haartsen et al., 1994), and boundary-element methods (Gerstoft and Schmidt, 1991). Various approaches for fluid-solid boundaries have been compared by Berman and Perkins (1990) and Berman (1991). The perturbation and Rayleigh methods are limited to surfaces with small root-mean-square heights and/or slopes. The boundary-integral and boundary-element methods are based on models of piecewise homogeneous media. 
These methods are slightly less general than finite-difference methods but for complex models the computational effort is comparable. The finite-difference method is unique in that it solves the scattering problem for pulses directly in the time domain rather than carrying out a Fourier synthesis of frequency-domain results.

Kuperman and Schmidt (1986) combined a boundaryperturbation method with a wave-number integral approach to study the effects of randomly rough surfaces on the propagation of coherent compressional and shear waves in a stratified fluid-solid media. They concluded that rough interface scattering into shear waves contributed significantly to the transmission loss for both shallow water and Arctic (icecovered) propagation. Dacol and Berman (1988) applied the extinction theorem method to scattering from a rough fluidsolid boundary. They pointed out the importance of transmission into the bottom (compressional and shear) as a loss mechanism and they identified peaks in the scattering coefficients at the compressional and shear critical angles. Dougherty and Stephen $(1988,1991)$, using the finitedifference method, and Kuperman and Schmidt (1989), using a boundary-perturbation approach (which was theoretically equivalent to Dacol and Berman's method), showed that secondary scattering into Stoneley (Scholte, interface) waves was a significant loss mechanism in addition to scattering into shear-body waves. Gerstoft and Schmidt (1991) used a boundary element approach to study scattering from surface and shallow buried "facets" and also identified the importance of interface and flexural waves as a significant loss mechanism.

\section{THE NUMERICAL SCATTERING CHAMBER (NSC)}

The name "numerical scattering chamber" refers to the concept of computing scattering functions numerically by the finite-difference method. The numerical scattering chamber concept consists of five components: (i) using the finitedifference method to obtain scattering solutions with the advantages outlined above, (ii) totally surrounding the scattering region with absorbing boundaries ("truncated domains"), (iii) insonifying the scattering region with a pulse beam, (iv) decomposing the scattered field into intercept time-angle space, and (v) computing scattering strength as a function of angle. Similar approaches have been taken by other authors. Thorsos and Jackson (1989) addressed scattering from a rough, pressure-release surface using an integral-equation method. Fricke (1991, 1993) studied rough surface scattering from ice keels using a point source rather than a pulse beam for the incident field. Levander et al. (1993) considered scattering from a rough seafloor using a semi-infinite plane wave for the incident field.

The chamber is completely surrounded by an absorbing region (Fig. 2). In the case described here we assume a twodimensional Cartesian coordinate system; however, the technique can be extended to three dimensions (Burns and Stephen, 1990). The structure inside the chamber can be completely arbitrary and is specified on a grid with typically 10-60 grid points per wavelength both vertically and horizontally. At each grid point we define the compressional and shear velocities, the compressional and shear attenuations,

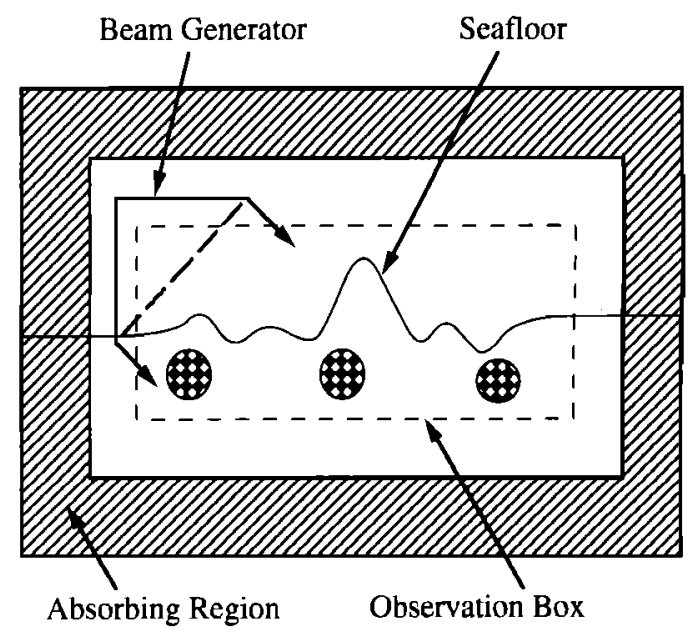

FIG. 2. The numerical scattering chamber, in two-dimensional Cartesian coordinates, is totally surrounded by an anechoic (absorbing) region. Inside the chamber one can "place" arbitrary-depth and range-dependent structure including interface and volume scatterers. These are defined by specifying compressional and shear velocity, compressional and shear attenuation, and density on a grid with from 10 to 60 grid points per wavelength. The structure is insonified by a beam generator that is transparent to the scattered energy. Time series of the total field (pressure for fluids and normalized dilatation and rotation for solids) are acquired on a box of receivers surrounding the scattering region. These time series are processed to obtain the scattered energy as a function of angle. Snapshots of compressional and shear wave ficlds can also be generated to study the scattering mechanisms. This figure is from Stephen and Dougherty (1993).

and the density. For the seafloor scattering problem, we place in the chamber realizations of seafloor structure that contain either interface roughness and/or volume heterogeneities. The upper part of the chamber is generally water and the lower part represents sea-bottom material such as sediments or basalts. The interface between the water and the seafloor continues into the absorbing regions on either side.

The scattering region is insonified using a pulse beam of a given width, a given angle of incidence, and a given time dependence. Pulse beams allow us to track energy propagation and multipathing in the scattering region. The pulsebeam generator is transparent to the scattered field.

Completely surrounding the scattering region we place a box of receivers. In the water column these measure the pressure field of the incident and scattered waves. In the bottom we process the displacement response at the receivers to give time series of the normalized dilatation and normalized rotation (see the Appendix). These correspond to compressionaland shear-wave effects, respectively. When the shear modulus vanishes the normalized dilatation equals the pressure.

Inside the numerical scattering chamber we compute solutions to the elastic-wave equation by the finite-difference method (Virieux, 1986). The scheme is based on secondorder, centered finite differences of the elastodynamic equations expressed in particle displacement. The initial conditions are that particle displacement and particle velocity are zero and the boundary conditions correspond to the Sommerfeld radiation condition on all sides. A complete review of absorbing boundary conditions in finite-difference schemes for wave equations is given by Cheng (1994). In this paper the absorbing boundaries are implemented by solving the telegraph equation in a region surrounding the chamber 
(Cerjan et al., 1985; Levander, 1985). The parameters for the telegraph equation are selected to minimize artificial reflections. We treat attenuation within the chamber using the Padé-approximant method (Day and Minster, 1984; Stephen and Swift, 1994).

\section{A. The implementation of Gaussian pulse beams}

Many treatments of scattering are based on the infinite plane wave as the incident field. However, in a time-space formulation as used here the infinite plane wave is ill-posed. Since infinite plane waves at non-normal incidence to the seafloor interact with the seafloor at all times, the start-up field (implemented as a time dependent boundary condition) would have to consist of semi-infinite reflected and transmitted plane waves as well as a semi-infinite incident wave. Furthermore, at subcritical grazing incidence the transmitted plane wave would become an evanescent wave in the seafloor. To avoid this problem we truncate the lower edge of the incident plane wave so that it does not hit the seafloor until after the initial time for the computation. By truncating the top edge of the plane wave as well we can take advantage of a great deal of analysis that has been carried out for beams (e.g., Červený et al., 1982; Thorsos, 1988). Unfortunately scattering results for incident beams become dependent on the shape of the beam (Berman and Perkins, 1990).

Point sources are easily implemented in time-domain finite-difference schemes, but it is awkward obtaining a narrow-angle, low-grazing-angle incident field from a point source without interference from head waves and diving waves (Stephen and Dougherty, 1993). Arrays of point sources generate realistic beams but they always have sidelobes, which will affect the observed scattered field, and attenuating the sidelobe energy will distort the main lobe. Stephen and Dougherty (1993) addressed this problem by adding attenuation to the water column directly below the source to reduce the high-grazing-angle energy incident on the seafloor. In this paper we have chosen to implement Gaussian beams as the insonifying field for the numerical scattering chamber because they have a finite width, they do not have sidelobes, and they represent energy propagating at a single angle, in homogeneous media, or ray parameter, in stratified media.

The purpose of the Gaussian pulse beam is to restrict the insonifying field to a single grazing angle, or at least to a narrow range of grazing angles. Since scattering creates a resultant field that contains all angles, if we are to analyze the scattering problem we need to simplify the insonifying field. This is the principal advantage of the infinite plane wave; however, the infinite plane wave has other problems as mentioned in the discussion in Sec. IV A. Thorsos and Jackson (1989) and Thorsos (1988) encountered similar problems in applying integral-equation methods to compute scattering cross sections and they also use incident Gaussian beams to guard against edge effects. In fact, direct comparisons between their integral-equation solutions and our finitedifference solutions can be made. The response to more realistic insonifying fields, which contain a broad range of grazing angles, can be determined once the response of the component grazing angles is known. The angular width of our incident Gaussian beam, determined by beamforming the incident field, is shown in Stephen and Dougherty (1993). Schmidt and Jensen (1986) also encountered similar problems in determining seafloor reflection coefficients and they discuss the limitations of the angular spectrum of beams.

For a Gaussian beam, the infinite plane wave is weighted along the wavefront with an exponential profile, $A \exp \left(-\zeta^{2} / L^{2}\right)$, where $A$ is the maximum amplitude of the beam, $\zeta$ is the distance along the wavefront from the center of the beam, and $L$ is the half-width of the beam (the width where the amplitude is $e^{-1}$ of the peak amplitude). However, all beams spread because of energy diffracting out of the side of the beam. For a given propagation distance $\left(s-s_{0}\right)$, there is a specific initial half width, $L_{M}^{\text {opl }}$, at the range $s_{0}$ that will give the narrowest beam at the range $s$. In homogeneous material, the minimum initial half-width is given by (C̈ervený et al., 1982)

$$
L_{M}^{\mathrm{opt}}=\left[\left(2 \nu_{0} / \omega\right)\left(s-s_{0}\right)\right]^{1 / 2},
$$

where $v_{0}$ is the propagation velocity and $\omega$ is the angular frequency. As path length increases, the minimum beam width increases.

The "spreading" of a Gaussian beam comes from the exact solution to the problem. Take initially an infinite plane wave in a homogeneous medium and then truncate it. You can truncate it sharply with a step function or smoothly with a Gaussian beam or any other way. By Huygen's principle (e.g., Pierce, 1989, pp. 174-175), the propagating wavefront, after truncating the initial plane wave, will be determined from the superposition of wavefronts from point sources on the initial truncated wave. These will contribute energy at angles other than the normal to the original plane wave. By analogy with diffractions of energy around the edges of a slit, which is also described by Huygen's principle, this spreading of the truncated plane wave can be referred to as "diffraction." This spreading is not an artifact of some approximation to the wave equation nor is it a result of the numerical method.

In bottom-interaction studies the footprint on the seafloor increases with smaller grazing angle and the distance over which a finite beam must propagate to cover this footprint also increases. So the narrowest possible beam width increases with decreasing grazing angle.

In the time dimension, the insonifying field is a pressure pulse with the shape given by the third derivative of a Gaussian curve [see Appendix E of Stephen et al. (1985)]. This waveform has the advantage that its spectrum is also a Gaussian curve. The half-power points of the pressure spectrum occur at $0.68 f_{p}$ and $1.36 f_{p}$, where $f_{p}$ is the peak frequency. The bandwidth of the source is one octave.

For example, we consider a Gaussian pulse beam propagating in a straight, constant-width channel in a homogeneous medium (Fig. 3). The channel corresponds to an idealized, finite-width "ray" and we can imagine the channel reflecting and refracting at a sharp interface according to Snell's law. (On refraction at a sharp interface the channel width would change.) At $15^{\circ}$ grazing incidence we take a channel width of $18.6 \lambda_{w}$ (where $\lambda_{w}$ is the wavelength in water, with a velocity of $1.5 \mathrm{~km} / \mathrm{s}$, at the peak frequency of 


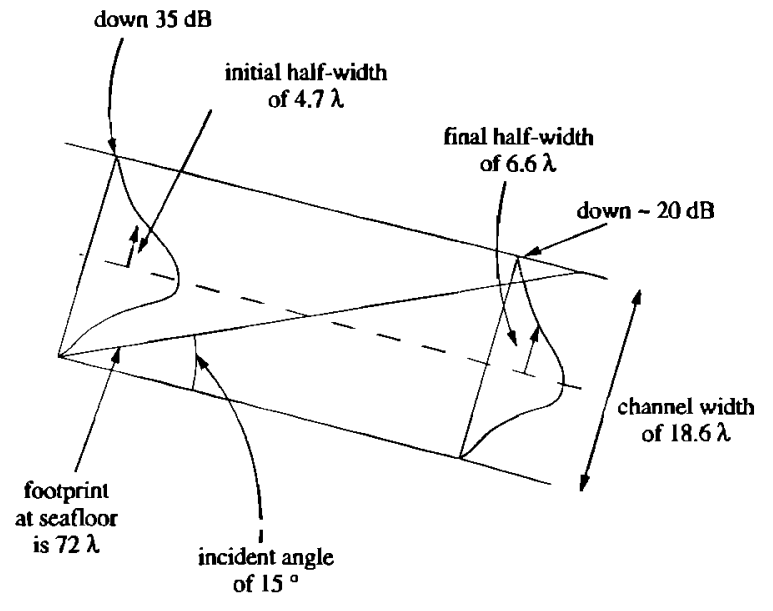

FIG. 3. Gaussian pulse beams are used to insonify patches of seafloor in the numerical scattering chamber. They have the advantage of providing localized energy at a fixed grazing angle without sidelobes. We can imagine a channel with parallel sides within which the pulse beam propagates. The intersection of this channel with the seafloor will be the footprint within which all bottom scatter occurs. We would like the footprint and the channel to be as narrow as possible in order to localize the bottom interaction and to minimize the size of the computational grid. However, for a given propagation distance there is a minimum width Gaussian beam (Cerveny et al., 1982). Consider a pulse beam incident on a horizontal plane (a virtual seafloor) at $15^{\circ}$ grazing angle. We further specify that the beam should be down at least $20 \mathrm{~dB}$ at the edges of the channel. The narrowest Gaussian beam that meets these criteria starts with a half-widih of $4.7 \lambda_{w}$ and ends with a halfwidth of $6.6 \lambda_{n}$. (All length scales are given in units of water wavelengths $\lambda_{n}$ at the peak frequency of the source pulse.) The smallest footprint on the seafloor that meets these criteria and is consistent with the wave equation is $72 \lambda_{w}$ wide. At $250 \mathrm{~Hz}$ the smallest footprint is $432 \mathrm{~m}$ wide.

the source in pressure) and its footprint on the horizontal plane is $72 \lambda_{w}$. The narrowest Gaussian beam that can propagate across the footprint starts with a half-width of $4.7 \lambda_{w}$ and is down $35 \mathrm{~dB}$ on the edges of the channel. After propagating across the footprint, the beam has spread to a half-width of $6.6 \lambda_{w}$ and the beam is down only $20 \mathrm{~dB}$ on the edges of the channel. This "spreading" of the beam is caused by diffraction of energy out of the ray tube and is not caused by geometrical spreading. For this model the edges of the ray tube are parallel. The spreading loss within the NSC caused by this effect is negligible. For example, the computed scattering strength of the specular reflection from a flat seafloor (the dashed line in Fig. 9) is $0 \mathrm{~dB}$ within $0.2 \mathrm{~dB}$.

Figure 4 shows snapshots of the pulse beam crossing the numerical scattering chamber for the case of a homogeneous medium. Parameters for the model are given in Table I. All snapshots from the NSC display compressional or shear "amplitude densities" as defined in the Appendix. To minimize the size of the computational domain the whole beam is never entirely within the chamber. The beam is over 18 wavelengths across and about 3 wavelengths long. There is no indication of reflections from the boundaries of the chamber.

For a low-grazing-angle problem there can be very many wavelength size scatterers within the footprint of the narrowest acceptable beam. Note that, although this issue is clearly evident in the time-space-domain finite-difference solutions, the physical constraints are placed by the wave equation and the physics of sound propagation. All modeling approaches and analysis techniques face the same limitations.

\section{B. Beamforming}

To quantify the amount of energy propagating at various angles, we apply beamforming to the time series results from the numerical scattering chamber. To represent fully the lowangle energy and the energy trapped near the interface, we include in the beamforming the time series on the vertical arrays on either side of the scattering chamber as well as on the horizontal array at the top of the chamber. In this discussion we only consider the pressure response in the water column and the energy scattered upward in the NSC. The downward-scattered compressional and shear waves in the bottom could be treated in a similar fashion using the dilatational and rotational fields.

Before applying the beamforming, we subtract the incident field, computed for a model with no structure in the chamber, from the observed time series (Fig. 4). This is consistent with the common definition in scattering theory that the scattered field is the total field minus the incident field (e.g., Pierce, 1989, p. 425).

Beamforming, or slant stacking, is an application of the radon transform (Chapman, 1978; 1981, Durrani and Bisset, 1984). The slant stack in geophysics and acoustics is normally applied to single linear arrays of receivers (Kappus et al., 1990; Stoffa et al., 1981). However, in the analysis presented here we need to represent accurately the lowgrazing-angle propagation and we use both vertical and horizontal linear arrays simultaneously in the slant stack. Our approach is similar to Fricke (1991), but, since there are differences from conventional processing, we outline briefly here the algorithm that we used.

The time series from the NSC represent the pressure field (normalized dilatation or normalized rotation, see the Appendix) as functions of time and range, $u(t, x)$, and time and depth, $u(t, z)$. To display the results, we use the ray parameter $p$, which is related to the incident angle $i$ and the grazing angle $\theta$ by $p=\sin (i) / V=\cos (\theta) / V$, where $V$ is the velocity along the array and it is assumed constant. The beam formed field in $\tau-p$ space is then given, in terms of the horizontal array data for example, by

$$
\tilde{u}(\tau, p)=\int_{\infty}^{\infty} u(t+p x, x) d x,
$$

where $\tau$ is the intercept time, that is, the time referenced to a trace at the origin $(x=0)$.

This transform is implemented for a finite amount of discrete data on orthogonal linear arrays as follows. The horizontal array consists of $M$ traces separated by $\Delta x$. The vertical arrays on the left and right sides extend from the horizontal array down to the seafloor. They consist of $N_{L}$ and $N_{R}$ traces, respectively, and the traces are separated by $\Delta z$. All traces have $K$ time points at an interval of $\Delta t$.

The NSC is constructed so that all scattering occurs within the receiver box. Outside of the receiver box, in the absorbing boundary regions, the structure is laterally homo- 

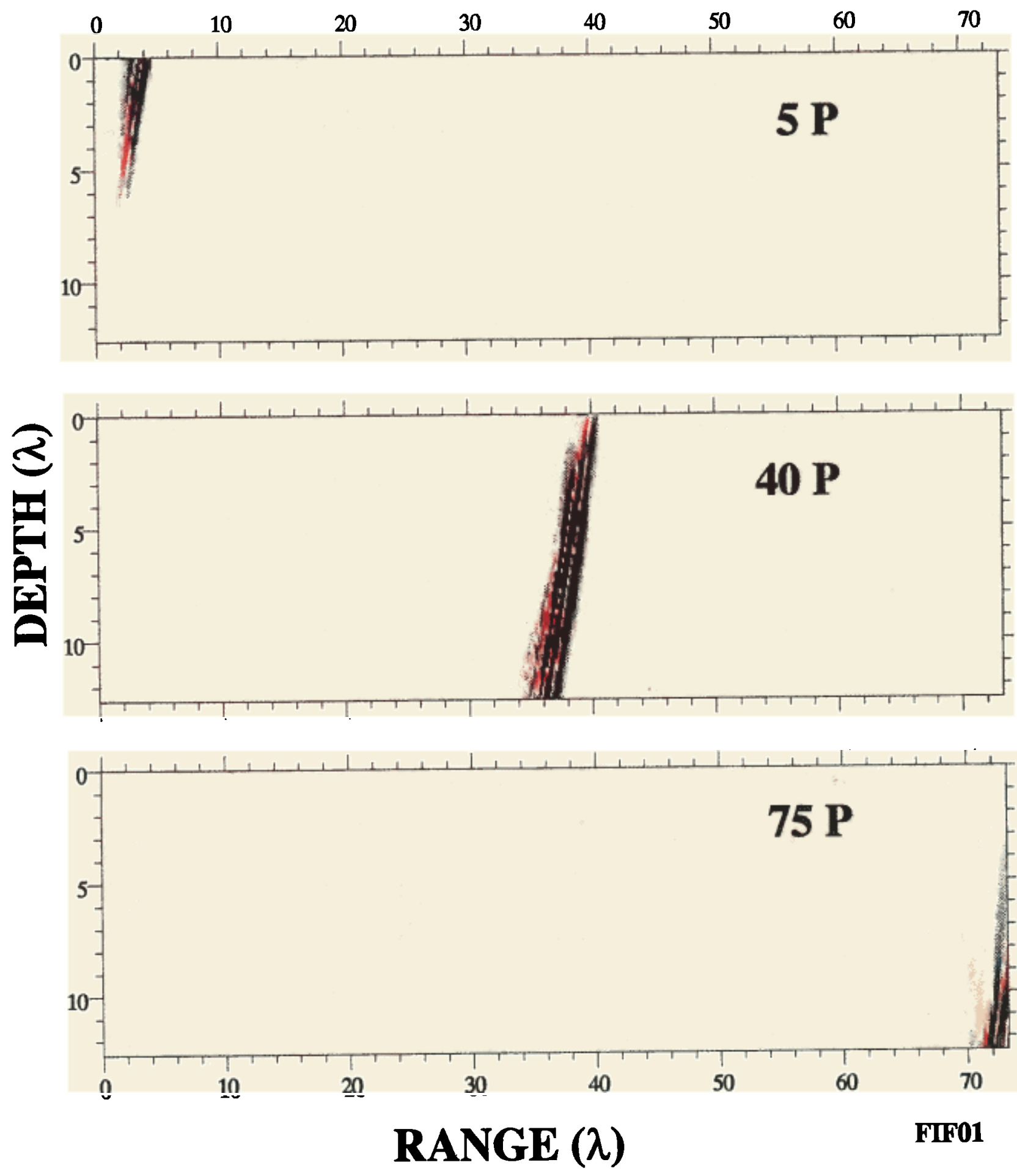

No Seafloor

Compressional

FIG. 4. Snapshots of the compressional wave field at times of 5,40 , and 75 periods $(P)$ are shown for the Gaussian pulse beam as it propagates across the numerical scattering chamber filled with homogeneous water. The beam is about three wavelengths long and over 18 wavelengths across. There are no discernible reflections from the absorbing boundaries. 


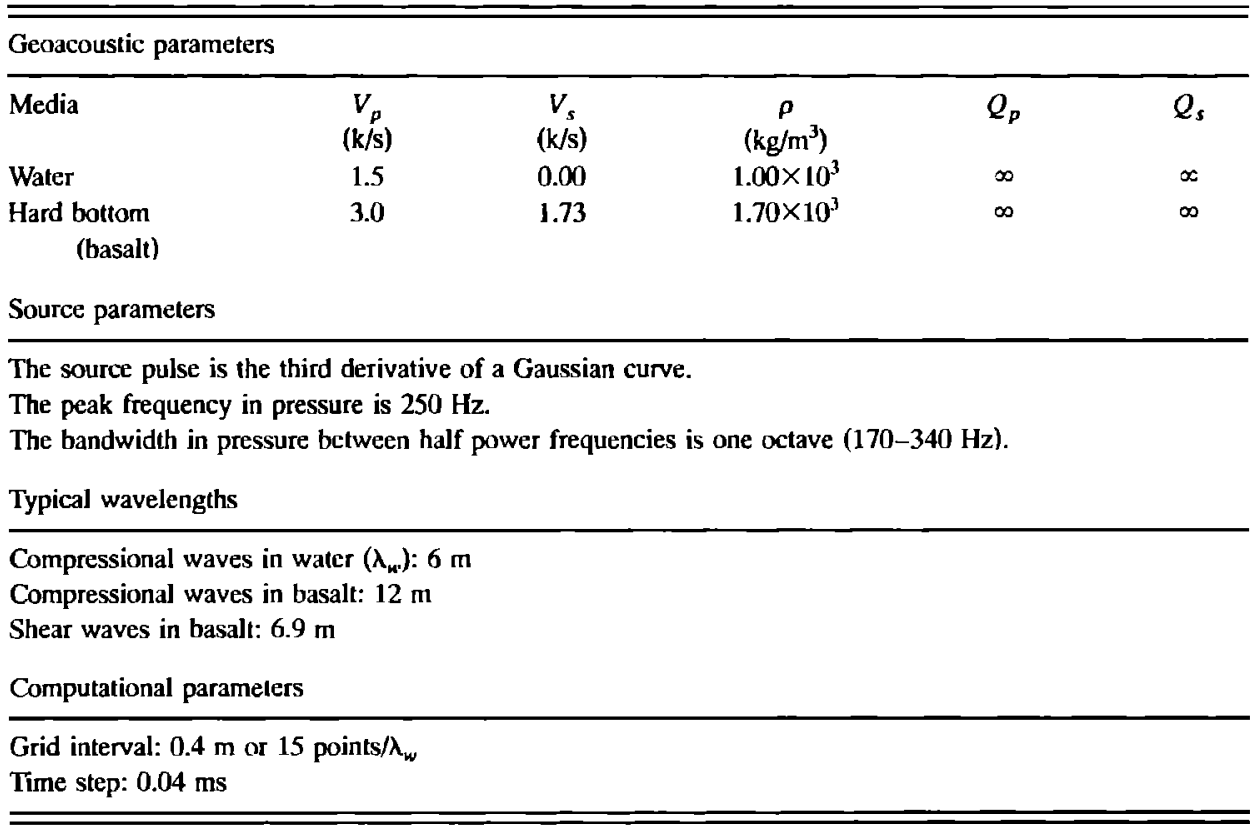

geneous so that there will be no scattering. For a given structure within the NSC, all backscattered energy in the water column, with grazing angles from $0^{\circ}$ to $90^{\circ}$, crosses the horizontal array and the left vertical array. All forward-scattered energy, with grazing angles from $90^{\circ}$ to $180^{\circ}$, crosses the horizontal array and the right vertical array. The following discussion applies to the backscattered beams but the forward-scattered beams are treated in an analogous fashion. For a given ray parameter or angle, the width of the wavefront intersecting the array is

$$
W_{L}=\left(N_{L}-1\right) \Delta z \sin i+(M-1) \Delta x \cos i .
$$

Time is referenced to the trace at the upper left corner and traces are labeled incrementally with distance away from the upper left corner. (Note that for the forward-scattered field, time is referenced to the trace at the upper right corner.) The slant stack, normalized to unit length along the wavefront, is

$$
\begin{aligned}
\tilde{u}\left(\tau_{j}, p_{k}\right)= & \left(\Delta z \sin i \sum_{l=2}^{N} u^{\mathrm{int}}\left[\tau_{j}+(l-1) q \Delta z, z_{l}\right]\right. \\
& \left.+\Delta x \cos i \sum_{l=1}^{M-1} u^{\mathrm{int}}\left[\tau_{j}+(l-1) p \Delta x, z_{l}\right]\right) \\
& \times\left(W_{L}^{-1}\right),
\end{aligned}
$$

where $q=\left(1-p^{2}\right)^{1 / 2}$. Linear interpolation is carried out between discrete time points. To avoid edge effects a cosine taper is applied within $W_{L} / 10$ from the edges of the wavefront. With this normalization, the same plane wave at different angles of incidence will have traces of the same magnitude in the slant stack.

The NSC output has a low level of high- and lowfrequency numerical noise that can reduce the sensitivity of the method to low levels of scatter. Since we know that the scattering process is linear and we know the bandwidth of the source, we filter out the numerical noise at parasitic fre- quencies. For example, for a source with $60 \mathrm{~dB}$ down points at 2 and $25 \mathrm{~Hz}$ we apply a bandpass filter between 1 and 30 Hz.

Various representations of scattering (scattering strength, scattering cross section, target strength) are based on ratios of intensity, which, for acoustic waves, are proportional to the mean square of the pressure time series. (The Appendix presents the compressional and shear intensities that apply in the solid.) To obtain scattering functions, we take the mean square level of the stacked traces as a function of ray parameter or angle, normalized to the mean square level of the incident beam. The duration taken for the mean square levels is the same for both the incident and scattered fields and it is large enough to include all of the scattered energy. The scattering functions are therefore based on total energy in the incident and scattered fields.

In summary, the steps involved in producing scattering functions from the numerical scattering chamber are as follows.

(a) Create a model of the seafloor by defining compressional velocity, shear velocity, compressional attenuation, shear attenuation, and density on a twodimensional grid. Fluids are represented by a shear velocity of zero.

(b) Run the finite-difference calculations in the numerical scattering chamber to get the pressure time series of the total field on a box array of receivers (along the top and down the left and right sides in the water). Snapshots and videos can be used to gain insight into the scattering mechanisms.

(c) Subtract the incident field from the pressure time series to obtain the scattered field and take a radon transform (slant stack) of the box array data to obtain the scattered pressure field in $\tau$-p space.

(d) Compute scattering coefficients as a function of angle by dividing the scattered field intensity (mean square 


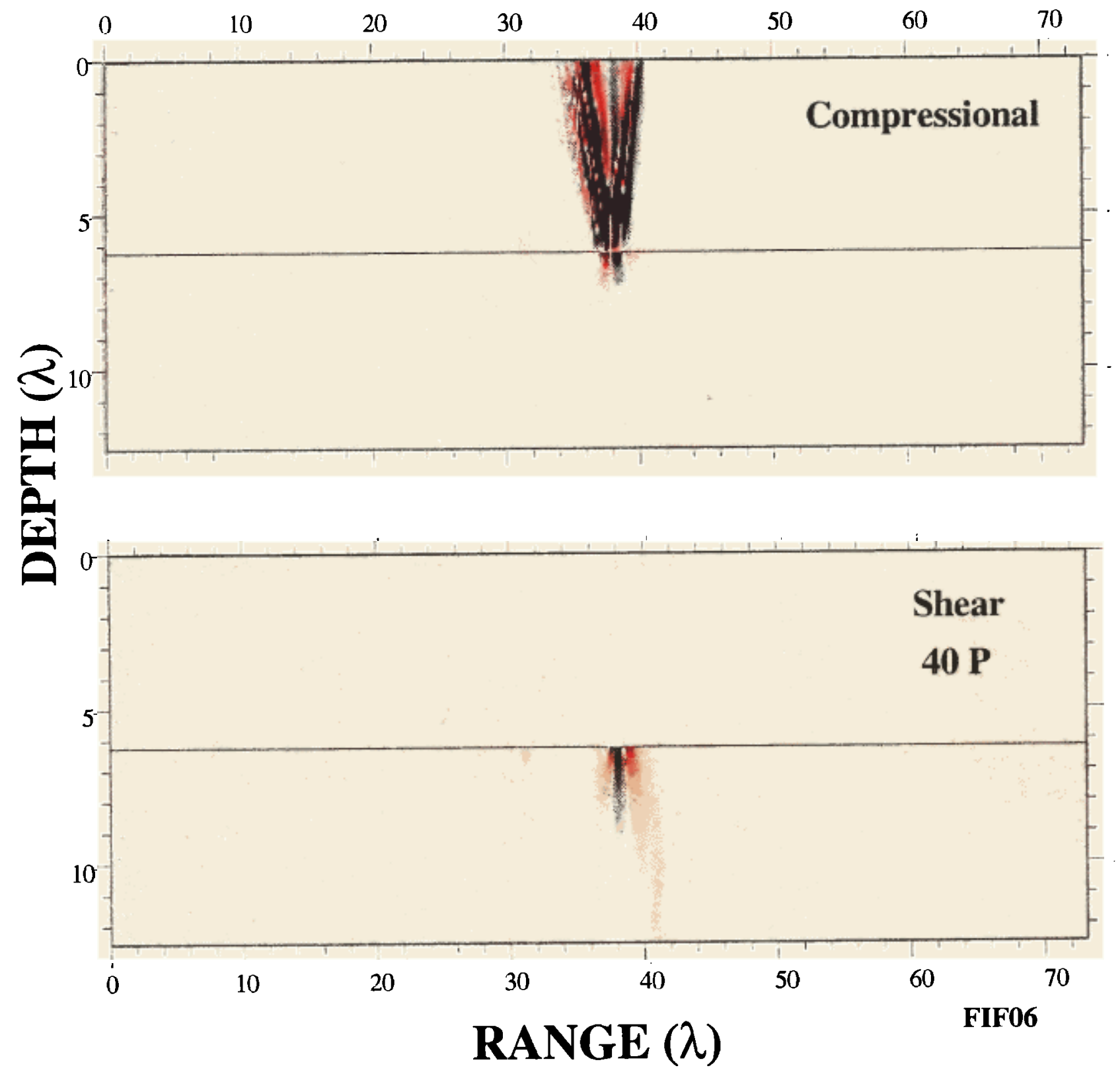

\section{Flat Seafloor \\ Hard Bottom \\ No Attenuation}

FIG. 5. Snapshots from the NSC for the compressional and shear wave fields at a flat water-basalt interface are shown at a time of $40 P$. (Model parameters are given in Table I.) Since the beam is incident at less than the critical grazing angle for compressional and shear waves, the beam is totally reflected. Evanescent "roots" of the reflected wave are observed below the seafloor in both the compressional and shear wave fields (Stephen and Bolmer, 1985).

level of the stacked traces normalized to beamwidth) by the intensity of the incident field.

\section{EXAMPLES}

\section{A. The scattering function for a single facet on the seafloor}

To demonstrate the numerical scattering chamber, we consider a single facet on a flat, hard bottom. As a reference we show first a snapshot from the NSC for a flat, hard sea- floor (Fig. 5). The incident beam angle $\left(15^{\circ}\right)$ is well below the critical grazing angles for compressional and shear waves in the bottom $\left(60^{\circ}\right.$ and $30^{\circ}$, respectively) and the beam is totally internally reflected. (The parameters for the models are given in Table I.) At this time step, the upper edge of the beam has not yet reached the seafloor and the lower edge of the beam has been reflected. These full-wave solutions also show the evanescent tails of compressional and shear energy in the bottom (Stephen and Bolmer, 1985). Note the absence of compressional and shear transmitted waves and compres-

R. A. Stephen and S. A. Swift: Numerical scattering chamber 


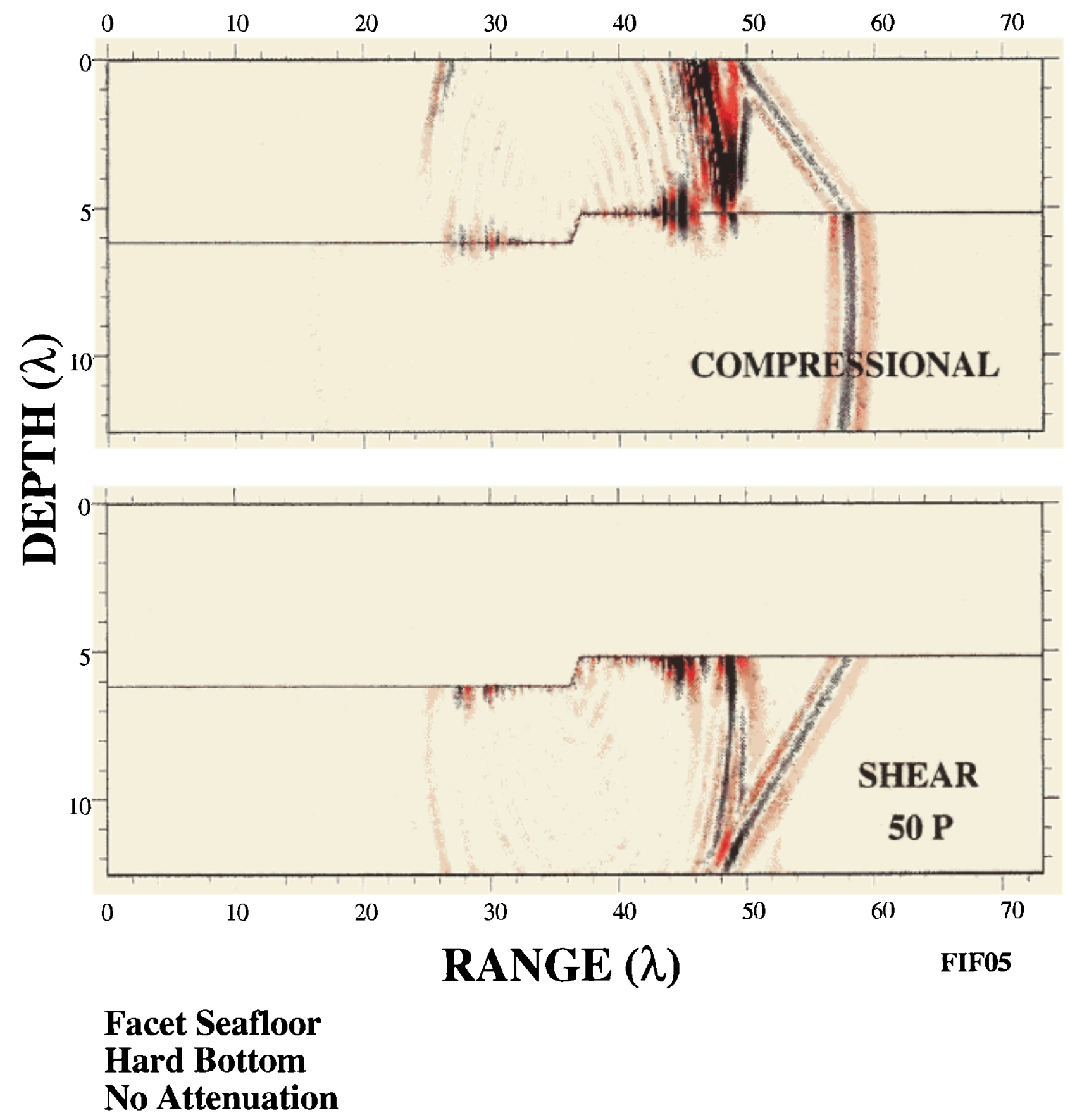

FIG. 6. Snapshots from the NSC for a single facet on the interface between water and basalt are shown at a time of $50 P$. The facet is one wavelength high and is inclined to be normal to the incident beam. The energy partitioning is quite complex. The facet acts as a secondary point source (diffractor) on the interface. It excites compressional and shear body waves in the bottom, a shear head wave in the bottom, a compressional head wave in the water, forward and backward propagating interface waves, and diffracted compressional and shear waves. The back-diffracted energy in the water column, which is significant for the long-range backscatter problem, exhibits time spread due to reverberation within the facet. There is both a magnitude and a duration in the backscattered field.

sional and shear head waves that would normally be present for a point source solution [see Fig. 7 of Dougherty and Stephen (1988)]. Since this flat seafloor model does not have any legitimate backscattered energy it can be used to determine the numerical noise floor of the method.

The wave field changes considerably when a single facet is introduced onto the seafloor (Fig. 6). In this case the facet is one wavelength high and its slope is normal to the incident beam. Because of its small size the facet acts as a point diffractor, or secondary source, on the seafloor after it is insonified by the incident beam. Compressional and shear transmitted waves and compressional and shear head waves are clearly observed in the forward direction. These are kinematically equivalent to the paths that would be expected for a point source on the seafloor. Because the facet is on the seafloor it also acts as a source of interface (Stoneley) waves. These are largest in the forward direction but they can be distinctly seen in the backward direction as well. Diffracted 


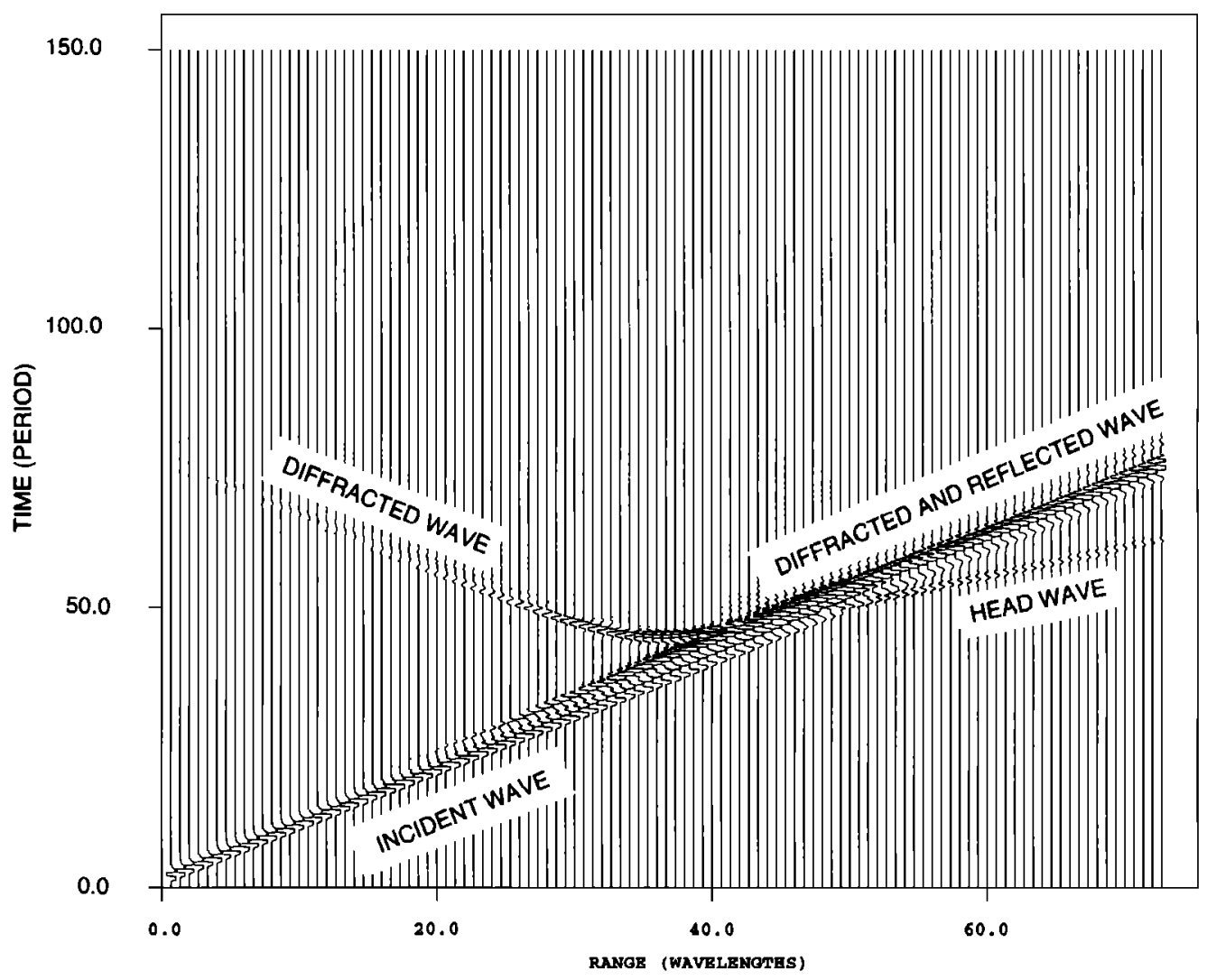

FIG. 7. Time series along a horizontal array in the water column for the single-facet model show the incident and reflected beams, the forward-propagating head wave, and the diffractions.

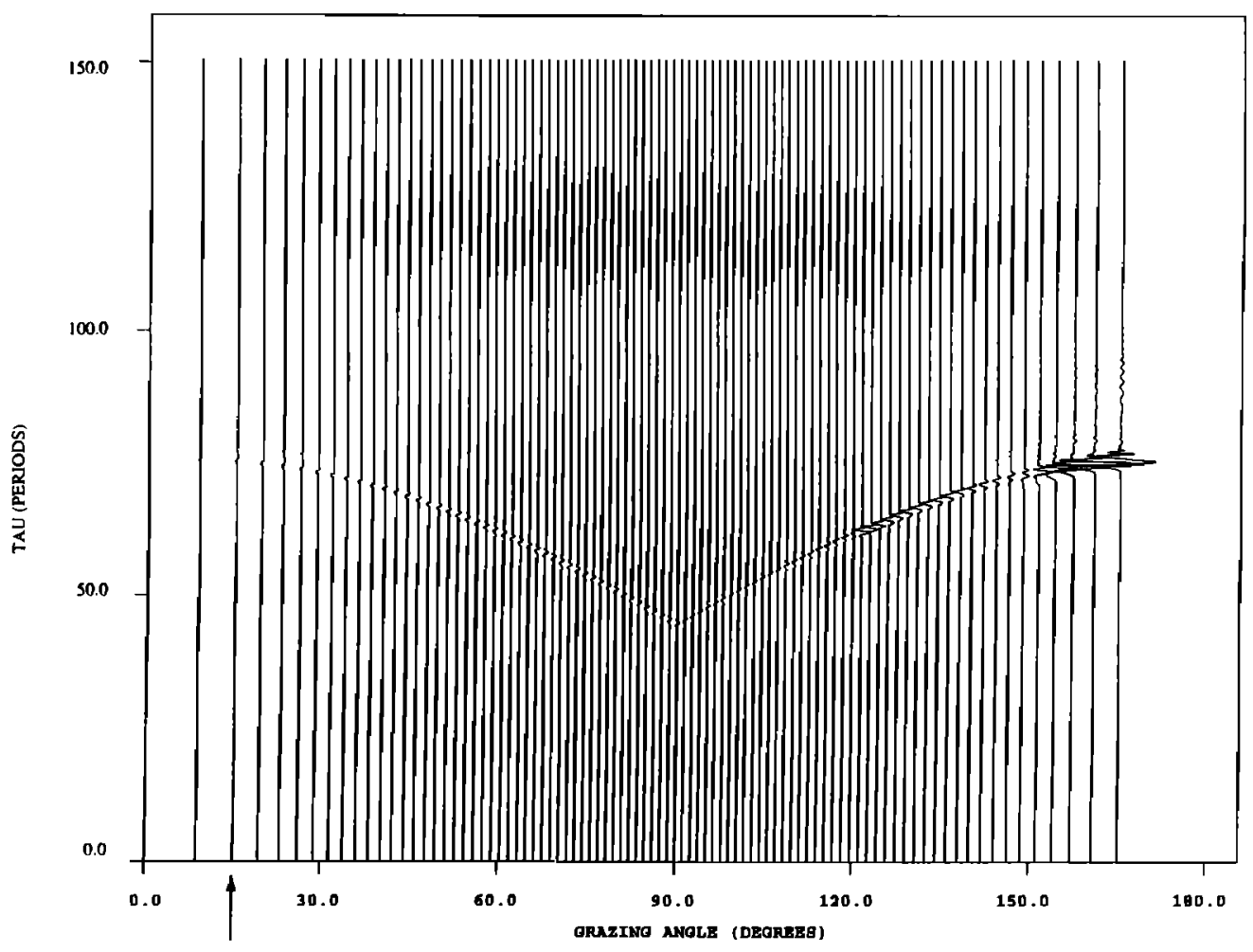

FIG. 8. Beamforming (or slant-stacking) the time series in Fig. 7 (after subtracting out the incident field) gives the time series of the "best" plane wave at various angles. The specularly reflected wave is the largest event and is observed around $165^{\circ}$. The forward-propagating head wave is the large event at $120^{\circ}$. The forward and backward diffractions have a uniform amplitude over a broad range of angles. Even though the facet was tilted to be normal to the incident beam there is no indication of a significant, normal incidence reflection back from the facet. This would be expected at the $15^{\circ}$ trace indicated by the arrow. Since the facet is only a wavelength in size it acts as a point diffractor. 


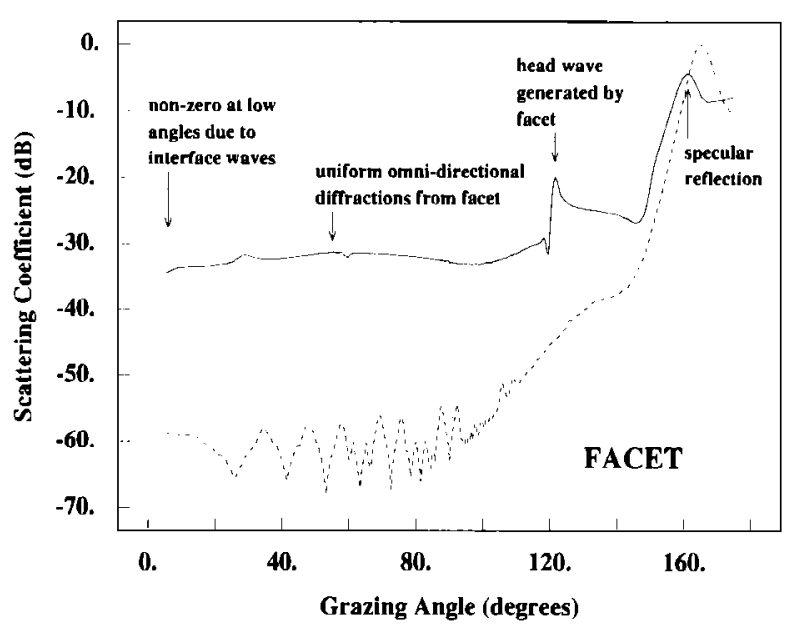

FIG. 9. The scattering coefficient as a function of angle is the energy of the $\tau-p$ field in Fig. 8 divided by the energy in the incident beam. The scattering function for the flat seafloor model (Fig. 5) is shown as a reference (dashed line). For the flat seafloor model the only arrival is the specular reflection with a peak value of $0 \mathrm{~dB}$. The width of the reflection peak indicates the resolution of the processing. Ideally it would be a delta function, but it has a finite width because of spreading of the beam in the NSC and because of the resolving power of the Fourier transform and slant-stacking procedure. The flat seafloor curve also shows the level of the numerical noise floor in the calculations. The scattering function for the facet model (solid line) shows the relatively uniform level of the diffracted field even at very low grazing angles (well above the numerical noise foor). The forward-scattered head wave is a significant event at $120^{\circ}$. The specular reflection for the facet model is lower in amplitude than for the flat seafloor model by about $6 \mathrm{~dB}$ because of energy lost in the scattering process.

compressional and shear body waves are also observed.

The time series for the facet model, on a horizontal row of receivers near the top of the model, are shown in Fig. 7. The incident beam, the reflected beam, the diffracted compressional wave, and the head wave can be clearly identified. The stacked traces are shown in Fig. 8. Events that appear as straight lines in the time series appear as points in the beamformed data. The incident and reflected beams, which are kinematically very close approximations to plane waves, both appear as straight lines in the $t-x$ data. The incident field is subtracted out prior to stacking, but on stacking the reflected field collapses to a point and appears as a concentration of energy at $165^{\circ}$ at a time of $75 P$ (Fig. 8). [The period $(P)$ is computed at the peak frequency of the source.] Also in the stacked data, the head wave appears as a concentration of energy at an angle of $120^{\circ}$ at about $60 \mathrm{P}$. The small-amplitude features across the whole section are omnidirectional diffractions from the facet.

Figure 9 shows the scattering function for the flat seafloor model (Fig. 5) and the facet model (Fig. 6). The backscattered energy for the flat seafloor model represents the numerical noise floor. The peak in the forward-scattered direction for the flat seafloor has a magnitude of $0 \mathrm{~dB}$ and represents the specularly reflected beam. The width of this peak represents the resolution of the incident beam and the postprocessing. For the facet the amplitude of the reflected beam is decreased. The offset of the seafloor at the facet creates a time shift in the reflected beam, which gives some destructive interference during stacking. The head wave shows up clearly at the forward critical angle, $120^{\circ}$. The diffracted energy is relatively uniform at a level of $-30 \mathrm{~dB}$.

\section{B. A rough, basaltic seafloor}

As a second example of the numerical scattering chamber we consider a rough, basaltic seafloor. This bathymetric profile is based on a canonical profile using seafloor statistics representative of the Pacific Ocean (Goff and Jordan, 1988, 1990). The profile was generated for a sample every $1.0 \mathrm{~m}$, but, we have applied the profile directly with a spacing of 0.4 $\mathrm{m}$. At the scale we have applied it, this profile is not the result of the Goff-Jordan algorithm, but it is still a good canonical, rough model for testing algorithms. The relief is about $10 \lambda_{w}$ over a range of $70 \lambda_{w}$. For a peak frequency of the source of $250 \mathrm{~Hz}$ this corresponds to $60 \mathrm{~m}$ of relief over $420 \mathrm{~m}$. In this example the bottom is completely homogeneous (Table I).

Figure 10 shows the compressional and shear wave fields in the scattering chamber 40 periods $(0.16 \mathrm{~s})$ after the pulse beam initiated contact with the seafloor. In the absence of the seafloor this frame would show a nearly vertical planar wavefront, $3 \lambda_{w}$ wide, at about $35 \lambda_{w}$ range. The rough seafloor has essentially destroyed the beam. Considerable energy is scattered into diffracted shear waves in the bottom and Stoneley waves on the interface. The interface waves propagate in forward and backward directions from the scattering centers until they reach another scattering center, where they reradiate back into the water column. Scattering centers in this context refers to topography on the seafloor that has enough curvature to act as a diffractor or secondary source. There are three broad humps, about $5 \lambda_{w}$ across, on the upward slope, at about $15 \lambda_{w}, 25 \lambda_{w}$, and $32 \lambda_{w}$, that are the source of distinct diffractions in the upward-scattered field (see below).

The weak energy in the compressional wave field between $42 \lambda_{w}$ and $60 \lambda_{w}$ includes the head wave energy and energy that was forward scattered as compressional waves in the bottom. In the shear wave frame the rightmost energy, beyond $42 \lambda_{w}$, is converted shear waves. The strong energy between $25 \lambda_{w}$ and $42 \lambda_{w}$ is scattered shear waves propagating downward and backward (to the left). Packets of high energy near the interface in both compressional and shear frames are interface waves generated by secondary scattering (Huygen's principle) from roughness elements on the interface. The backscattered shear and interface waves convert back into compressional waves in the water (seen in the compressional frame to the left of $30 \lambda_{w}$ ) and contribute to the low-angle backscattered field.

The backscattered field is quite complex in the time series plots (Fig. 11) where it appears as energy with negative slopes. The corresponding beamformed field (Fig. 12) is simpler and three distinct diffractions, from the humps mentioned in Fig. 10, are responsible for most of the upwardscattered field. At angles less than about $50^{\circ}$, the scattered field is more diffuse and has much longer time spreads (up to $50 P$ ) than the higher angle field. The scattering function for the very rough seafloor (Fig. 13) increases significantly at low angles and is about $6 \mathrm{~dB}$ greater at $15^{\circ}$ than at $90^{\circ}$. The monostatic backscatter value at $15^{\circ}$ is $-18.5 \mathrm{~dB}$. The in- 


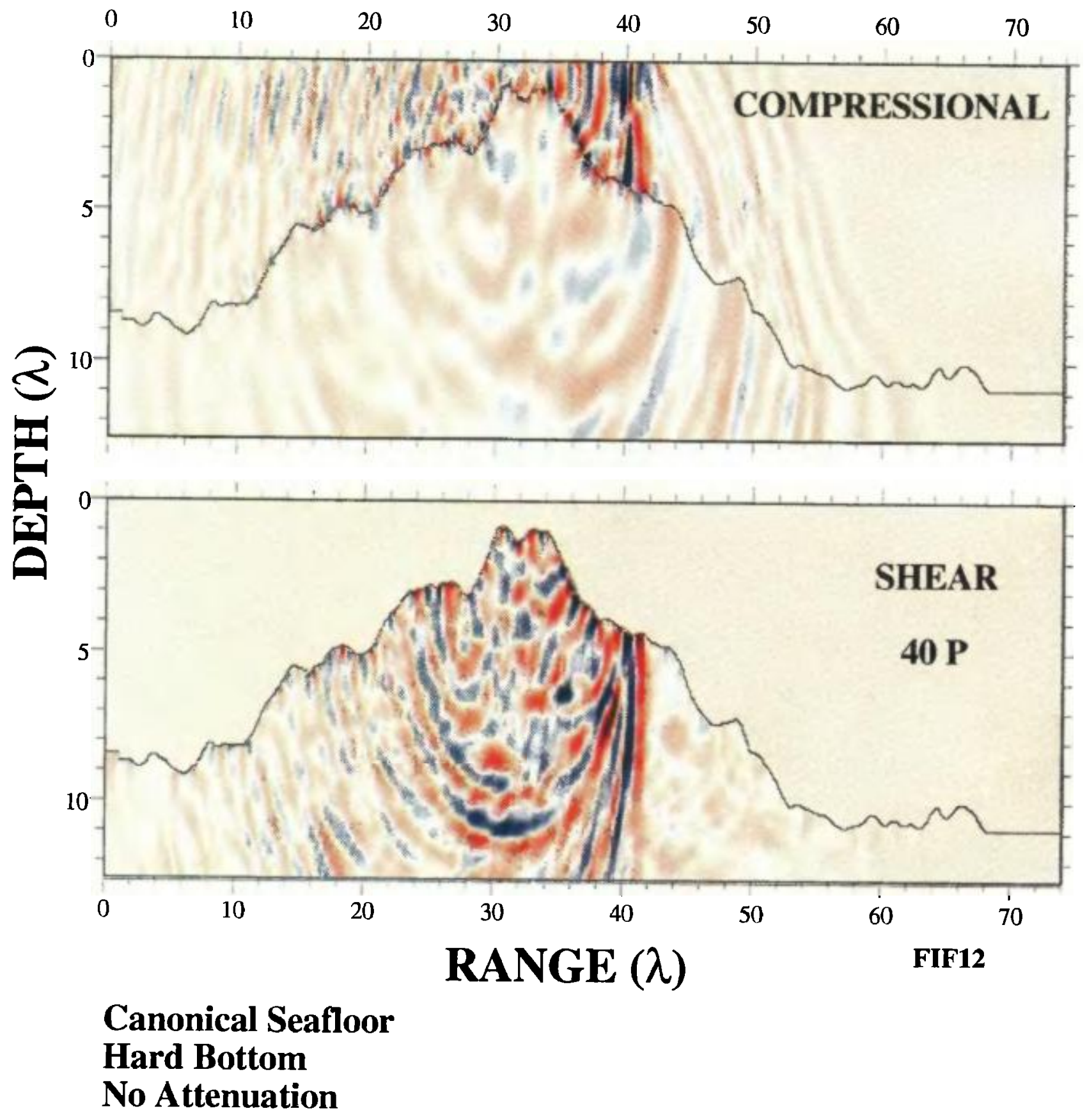

FIG. 10. Compressional and shear wave fronts are shown 40 periods after a pulse-beam at $15^{\circ}$ grazing incidence hit a very rough, basaltic seafloor from the left. If the seafloor were not present the beam would have propagated cleanly to about $35 \lambda_{w}$. Energy to the left of $35 \lambda_{w}$ is caused by backscatter and reverberation from the rough seafloor. Strong backscattered shear and interface waves can be seen in the shear wave frame. These convert back into compressional waves in the water and are a significant mechanism for strong, low-angle backscalter. (Model parameters are given in Table I.) This figure is from Stephen (1993).

crease in scattering cross section at low angles is consistent with previously reported results by Dougherty and Stephen (1988), Stephen and Dougherty (1993), Stephen et al. (1993), and Stephen (1993).

\section{DISCUSSION}

\section{A. Plane waves, beams, and the insonification pattern} on the seafloor

Many approaches to seafloor scattering problems are based on the notion of plane waves. Ideally the wavefront for a plane wave extends infinitely in the plane and has constant amplitude. This notion is particularly powerful in the analysis of energy partitioning at flat interfaces (e.g., Lamb's problem) and in the plane-wave decomposition of the field from a point source (e.g., the Sommerfeld and Weyl integrals). The Fourier, Hankel, and radon transforms used in these approaches are rigorously defined mathematical operations that are applied to general time series and fields independently of the physics of the underlying wave equations. However, although it is sometimes convenient to interpret the trans- 


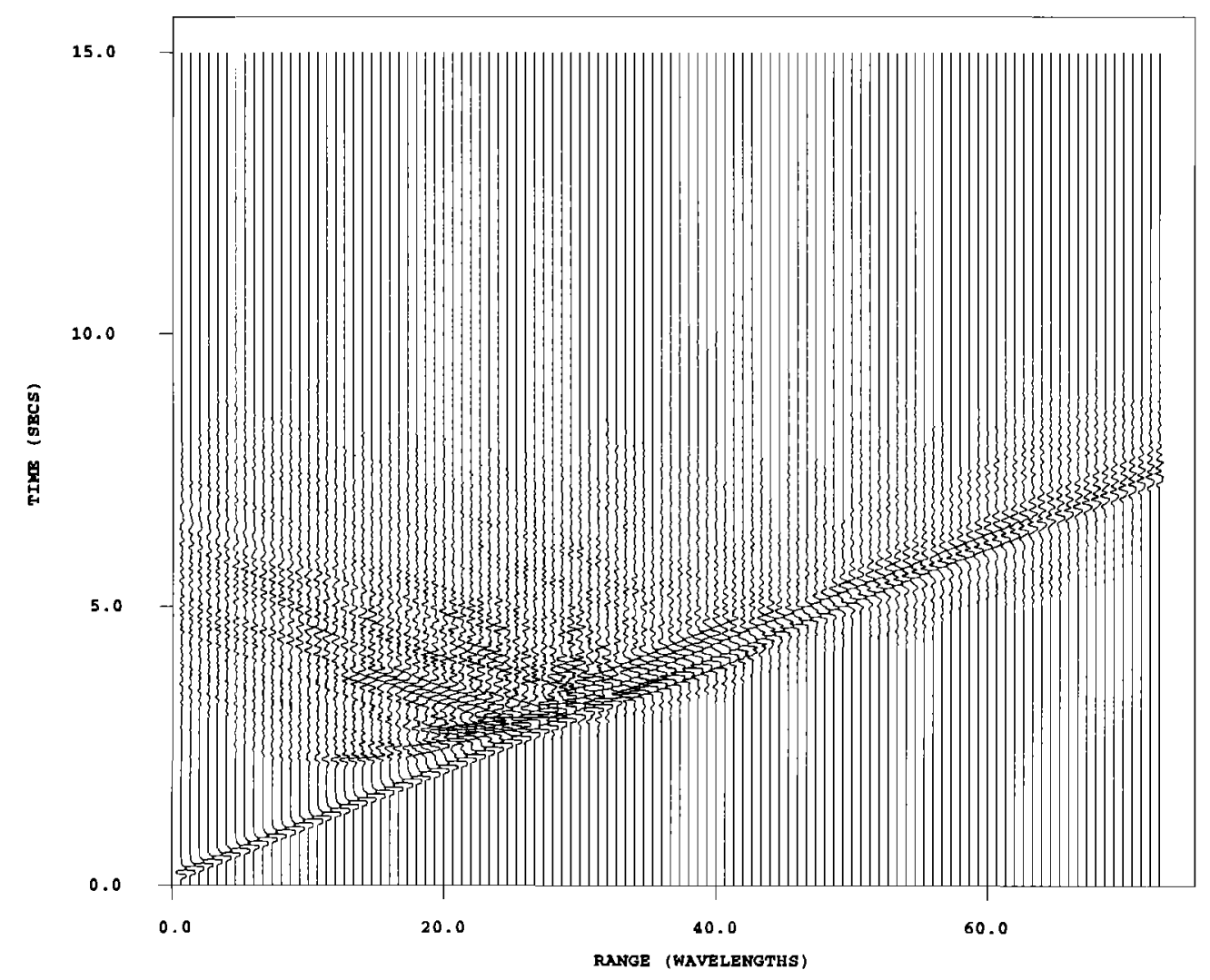

FIG. 11. Time series of pressure along a horizontal line of receivers are shown for the rough seafloor model of Fig. 10. To the right of the figure some weak energy occurs before the reflected beam. This is head wave energy created from a number of scattering centers and is analogous to the head wave energy in Fig. 7, but is less distinct. Similarly there is considerable backscattered energy behind the incident beam to the left of the figure. This corresponds to backscattered diffractions from a number of interacting scattering centers.

formed wave fields in terms of plane waves, it is important to remember that an infinite plane wave is not a valid source for the wave equation for heterogeneous media. We often lapse into thinking about the "incident plane wave" and the "scattered plane waves in the far field" but these are not valid entities in the context of the wave equation for heterogeneous media.

At the other extreme, it is also often convenient to imagine energy propagating along infinitely thin tubes or rays. For example, propagation from the source to the seafloor is often represented as an infinitely thin ray and the scattering from the seafloor is based on the notion of an infinitely wide planar wavefront (e.g., Caruthers and Bourgeois, 1992). Both the ray and the plane wavefront are physically unrealistic but they are convenient because they separate out the angle dependence of the interaction. Given the complexity of the wave field from a point source (spherical wavefront) above a flat interface the simplicity of single-angle interaction is well worth pursuing.

Synthesizing beams from arrays of point sources is a physically realistic and well-posed approach. However, it has the problem of sidelobes and results in beams almost as wide as the Gaussian beam approach. For example, a continuous line array, with a rectangular aperture function with the same half-width as the Gaussian beam in Fig. $3\left(4.7 \lambda_{w}\right)$, would have a beam width at the 20-dB level at the end of the channel of $13.4 \lambda_{w}$ (compared to $18.6 \lambda_{w}$ for the Gaussian beam) but the largest sidelobe would only be down $13.3 \mathrm{~dB}$ from the peak (Burdic, 1991). In strong scattering problems the sidelobes will excite scattering in the seafloor, which can reradiate at the same time and angle as scattering from the main beam.

The Gaussian beam approach is an attempt to improve on geometrical ray theory for finite-bandwidth problems and is a reasonable compromise (e.g., Thorsos and Jackson, 1989). It is a well-posed source to the wave equation and the energy is focused along a narrow range of angles.

It does not make sense to base a scattering theory on a plane-wave approximation in the far field but to ignore the propagation effects between the far field and the scattering region. In applied ocean acoustics the "beam" is created by an array of point or directional sources and the propagation effects from the source to the seafloor can be quite complex and can include caustics. Identifying the insonification pattern on the seafloor even at a single frequency is a challenging problem. Since the insonification pattern is an interference effect, not simply a ray or beam propagation effect, small changes in frequency can cause large changes in the pattern.

Also, since the beam is created by an array, reciprocity cannot be used to return the energy to the source-receiver location. For example, in Fig. 1, a monostatic experiment would have a receiver array at the same location as the source array. From the figure it is tempting to assume that the transmission loss along the ray path will be the same for propagation from the source to the seafloor as for propaga- 


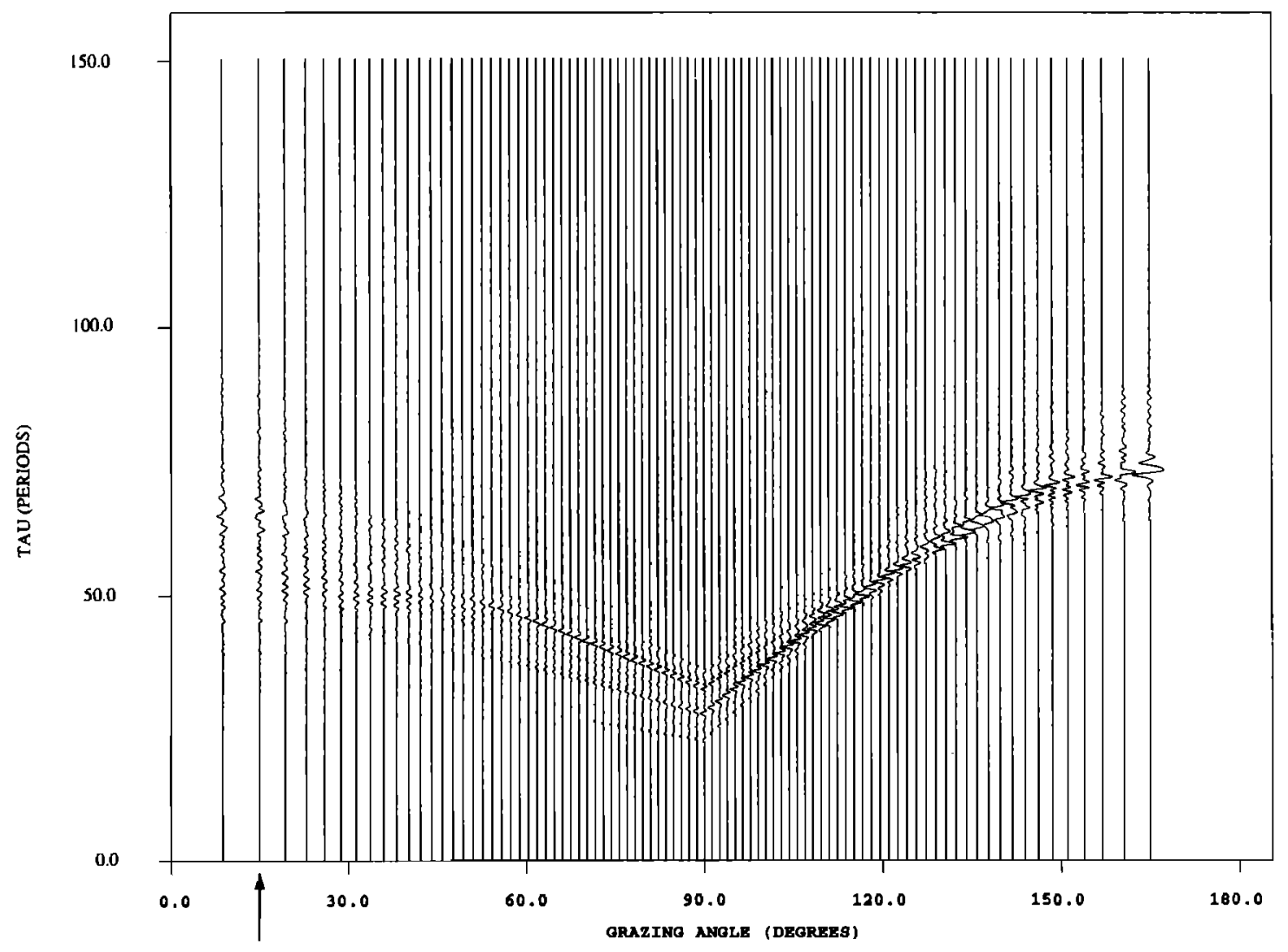

FIG. 12. The slant stack of the time series in Fig. 11 for the rough seafloor model is more complex than for the single facet (Fig. 8). At near-normal angles there appear to be three distinct diffractors, corresponding roughly to the three peaks on the upward slope of the seafloor (Fig. 10). These diffractions are less distinct at low angles. At low angles in both the forward and backward directions the duration, or time spread, of the reverberation increases considerably.

tion from the seafloor back to the source location. In this view, all we need is a single monostatic backscatter coefficient for the seafloor interaction. However reciprocity in acoustics works point-to-point not point-to-array. For a numerical demonstration of this fact see Figs. 14(a) and 16(a) in Stephen et al. (1985). If the beam is generated by an array,

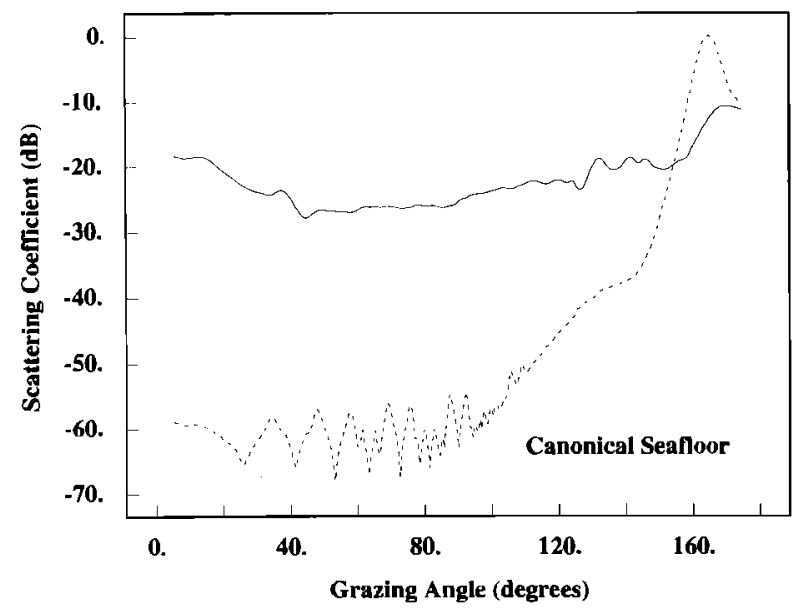

FIG. 13. The scattering function for the very rough seafloor model is remarkably flat. The effect of the rough seafioor is to take energy out of the specular direction and distribute it more or less uniformly across a range of angles. The backscattered energy (per unit area) increases significantly at low angles. the transmission loss on the return path is not the same as on the outgoing path.

Problems like these are prompting investigators to consider full-wave or total-field solutions to seafloorreverberation problems ( $F$. Tappert, personal communication). High returns from a given spot on the seafloor, in either a continuous wave or a broadband sense, may occur because of anomalies in the insonification pattern rather than because of features of the seafloor itself. Because of the complexity of the insonification pattern, it may be unreasonable to separate the propagation from the scattering using an angledependent function of any kind (based on plane waves, beams, etc.). A study should be carried out to evaluate the efficacy of an angle-dependent approach in the full field context.

\section{B. Potential applications of the numerical scattering chamber}

An important issue in seafloor scattering is the trade-off between deterministic and statistical representations of the seafloor geology (topography, volume heterogeneities, geoacoustic parameters, etc.) and the acoustic field. We need guidelines on how to separate the two effects. The numerical scattering chamber can be used to address a number of questions regarding this issue.

Dougherty and Stephen (1988) statistically chose the elastic parameters of the seafloor but generated the synthetics 
in a deterministic fashion. The coda and coherence of the acoustic field were represented by rms signal levels and covariance. Even if the geology of the seafloor were known deterministically at all scales, at some scale it would be reasonable to interpret the acoustic field stochastically. The scattered field from a number of seafloor models with the same statistical properties could be computed to confirm that the statistical properties of the acoustic field do not vary. Different stochastic representations of the seafloor (Gaussian, selfsimilar, etc.) could be treated to see how the statistics of the acoustic field varies.

At some fine scale, aspects of the geology will only be known stochastically. For example, volume heterogeneities could be represented by the mean and standard deviation of the geoacoustic properties of the sediments and crust. Given a stochastic representation of the geology, how can we create a stochastic representation of the acoustic field?

Other issues that can be addressed by applications of the numerical scattering chamber include (a) scattering from facets with a range of shapes, (b) scattering from canonical seafloors, (c) interface versus volume scattering in both sediment and basement, (d) interpolation methods, (e) bathymetric resolution requirements, (f) effects of intrinsic attenuation, (g) effects of sediment cover, (h) effects of shear properties in both sediments and basement, (i) 2-D versus 3-D scattering, (j) identification of scattering mechanisms, and (k) distinguishing scattering effects for swept frequency versus pulse sources. These issues will be covered in future papers.

\section{CONCLUSIONS}

Many problems in bottom-interacting ocean acoustics require a knowledge of the scattered field from a rough, laterally heterogeneous seafloor. Recent studies (Dougherty and Stephen, 1988; Dougherty and Stephen, 1991; Kuperman and Schmidt, 1989; Levander et al., 1993; Orcutt et al., 1993; Stephen, 1993) have shown that the physics of seafloor scattering can be quite complex with energy converting from compressional waves in the water to shear and interface waves at the seafloor and then reradiating as compressional waves back into the water. To investigate the physical mechanisms of scattering further and to quantify the magnitude, time spread, and angle spread of the scattered field we have developed a numerical scattering chamber based on the finite-difference solution to the two-way elastic (anelastic) wave equation.

The finite-difference method provides the capability to study full wave effects at the seafloor in range-dependent environments. The approach is particularly useful for pulse sources, for strong backscattering, and for studies of the response at and below the seafloor. Calculations are carried out in the time domain and solutions for a given source pulse are obtained directly. [The method can also obtain a continuouswave solution simply by using a continuous-wave source and running the computations until steady state is reached (e.g., Stephen, 1990).] Solutions are obtained for both forwardscattering and backscattering including conversions to shear and interface waves. Multiple interactions between scatterers are also completely included. Since the finite-difference method treats the whole model as a discrete grid, vertical and horizontal displacements (or velocities or accelerations) at the seafloor and within the bottom are obtained at the same time as the pressure field in the water. Insight into multipathing and bottom and sub-bottom scattering is obtained directly.

At a very rough, basaltic seafloor, conversion of energy from compressional waves in the water to shear and interface waves at the seafloor is an important physical mechanism for generating strong backscatter. Specular reflection from steep cliffs is not necessary to create strong backscatter. An unsedimented rough basalt has stronger backscatter at low grazing angles than at near normal angles. The duration, or reverberation, of the scattered field is also significantly greater at low angles. For the example of a rough seafloor considered in this paper, the backscatter cross section for a beam incident at $15^{\circ}$ grazing angle is $18.5 \mathrm{~dB}$ in the monostatic direction. This is about $6 \mathrm{~dB}$ greater than the scattering cross section directly upward (a grazing angle of $90^{\circ}$ ) for the same incident beam.

Representing the backscattered field by a single, angledependent coefficient is an oversimplification. Coherence of the scattered field before stacking and the time spread of the stacked (or beamformed) field are significant issues. In a strong scattering environment an angle-dependent separation of the wave field may not be valid and a full-field approach may be necessary.

\section{ACKNOWLEDGMENTS}

This work was carried out under support from the Office of Naval Research Acoustic Reverberation Special Research (Grant Number N00014-90-J-1493). Woods Hole Oceanographic Institution Contribution Number 8714.

\section{APPENDIX: ENERGY DENSITIES AND INTENSITIES FOR ACOUSTIC AND ELASTIC WAVES}

The mathematical treatment of sound propagation in fluids and solids is represented by the acoustic and elastic wave equations, respectively. However, even though the physics of acoustic propagation in compressible, nonviscous fluids is included in the elastic wave equation, the traditional derivations of the two equations are quite different. For most acoustic and seismic applications this distinction is irrelevant, but for seafloor-interaction problems, where acoustic and elastic effects are coupled, it is important to reconcile the two approaches. In this appendix, we present the concepts of energy density and intensity for both acoustic and elastic fields and show that they are consistent. The output of the numerical scattering chamber is given in terms of energy density and intensity for some applications and it is worthwhile to clearly state how these are defined so that results can be integrated and/or compared with other methods.

The energy density $w$ is the amount of energy in a wave at a given position and time and it is the sum of the kinetic and potential energy. For an acoustic wave the energy density $w_{\text {ac }}$ is (Morse and Feshbach, 1953, p. 309)

$$
w_{\mathrm{ac}}=\frac{1}{2} \rho \nu^{2}+\left(1 / 2 \rho c^{2}\right) p^{2},
$$


where $\rho$ and $c$ are the density and sound speed of the medium, respectively, $\nu$ is the magnitude of the field (or particle) velocity, and $p$ is excess pressure. For elastic waves the energy density $w_{\mathrm{el}}$ is (Morse and Feshbach, 1953, p. 150)

$$
w_{\mathrm{el}}=\frac{1}{2} \rho\left(\left|\frac{\partial \mathrm{s}}{\partial t}\right|\right)^{2}+\frac{1}{2}|\mathfrak{T} \cdot \mathfrak{S}|,
$$

where $\mathbf{s}$ is particle displacement, $\mathfrak{T}$ is the stress dyadic, and $\mathfrak{S}$ is the strain dyadic.

For a plane wave propagating in the $\mathbf{x}$ direction in a homogeneous medium, the acoustic energy density reduces to (Morse and Feshbach, 1953, pp. 311-312 with some algebraic manipulation)

$$
w_{\mathrm{ac}}=\rho\left[g^{\prime}(x-c t)\right]^{2},
$$

where $g(x-c t)$ is the velocity potential and $\mathrm{i} g^{\prime}(x-c t)$ is the particle velocity. (The unit vectors in the Cartesian directions $x, y$, and $z$ are $\mathbf{i}, \mathbf{j}$, and $\mathbf{k}$.) In a homogeneous elastic medium the solution to the wave equation separates into compressional (longitudinal) and shear (transverse) parts. For a plane wave propagating in the $x$ direction, the compressional and shear energy densities are (Morse and Feshbach, 1953, p. 150)

$$
\begin{aligned}
& w_{\text {comp }}=(\lambda+2 \mu)\left[f^{\prime \prime}\left(x-c_{c} t\right)\right]^{2}, \\
& w_{\text {shear }}=\mu\left[F^{\prime \prime}\left(x-c_{s} t\right)\right]^{2},
\end{aligned}
$$

where $\lambda$ and $\mu$ are Lamé parameters ( $\mu$ is the shear modulus), the scalar displacement potential is $f\left(x-c_{c} t\right)$, and the vector displacement potential is $\mathbf{A}=\mathbf{j} F\left(x-c_{s} t\right)$. The compressional and shear wave speeds are

$$
c_{c}=\sqrt{(\lambda+2 \mu) / \rho}, \quad c_{s}=\sqrt{\mu / \rho} .
$$

The particle velocities corresponding to the compressional and shear waves are $-\mathbf{i} c_{c} f^{\prime \prime}\left(x-c_{c} t\right)$ and $-\mathbf{k} c_{s} F^{\prime \prime}\left(x-c_{s} t\right)$, respectively. If the shear modulus vanishes, no shear waves are supported, $\lambda$ reduces to the bulk modulus, and the compressional wave speed reduces to the acoustic wave speed. In this case, $g^{\prime}\left(x-c_{c} t\right)=-c_{c} f^{\prime \prime}\left(x-c_{c} t\right)$ and the compressional energy density reduces to the acoustic energy density.

It is often convenient to express the compressional and shear energy density in terms of the divergence and curl of the displacement field (e.g., Dougherty and Stephen, 1988). [(Aki and Richards, 1980), pp. 64-77) discuss the separation of the elastic wave equation into two wave equations in terms of the compressional and shear potentials.] For compressional waves, the scalar displacement potential $\phi$ by definition satisfies the relation $\mathbf{u}=\boldsymbol{\nabla} \phi$. It also satisfies the wave equation (Morse and Feshbach, 1953, p. 142)

$$
\frac{\partial^{2} \phi}{\partial t^{2}}=c_{\mathrm{c}}^{2} \nabla^{2} \phi
$$

which has a solution (as assumed above) of $\phi=f\left(x-c_{\mathfrak{c}} t\right)$. By substituting the solution $f$ into the left-hand side and expanding the right-hand side, we have

$$
f^{\prime \prime}\left(x-c_{c} t\right)=\boldsymbol{\nabla} \cdot \boldsymbol{\nabla} \phi=\boldsymbol{\nabla} \cdot \mathbf{u} .
$$

Similarly for shear waves the vector displacement potential A satisfies $\mathbf{u}=\boldsymbol{\nabla} \times \mathbf{A}$ and the wave equation (Morse and Feshbach, 1953, p. 143)

$$
\frac{\partial^{2} \mathbf{A}}{\partial t^{2}}=-c_{s}^{2} \boldsymbol{\nabla} \times \boldsymbol{\nabla} \times \mathbf{A}=c_{s}^{2} \nabla^{2} \mathbf{A}
$$

where we have used the identity

$$
\boldsymbol{\nabla} \times \boldsymbol{\nabla} \times \mathbf{A}=\boldsymbol{\nabla}(\boldsymbol{\nabla} \cdot \mathbf{A})-\nabla^{2} \mathbf{A}
$$

and the property of $\mathbf{A}$, by definition, that $\boldsymbol{\nabla} \cdot \mathbf{A}=\mathbf{0}$. The vector wave equation has the solution (as assumed above) of $\mathbf{A}=\mathbf{j} F\left(x-c_{s} t\right)$ and

$$
F^{\prime \prime}\left(x-c_{s} t\right)=\nabla \times \mathbf{u} .
$$

The compressional and shear energy densities can then be expressed as

$$
w_{\text {comp }}=(\lambda+2 \mu)[\nabla \cdot \mathbf{u}]^{2}, \quad w_{\text {shear }}=\mu[\nabla \times \mathbf{u}]^{2} .
$$

Field intensity is the same as the density of energy flow (Morse and Feshbach, 1953, p. 320), the flux rate of energy (Aki and Richards, 1980, p. 127), or the transmitted power per unit area (Morse and Feshbach, 1953, p. 151). The acoustic field intensity $S_{a c}$ is (Morse and Feshbach, 1953, pp. 312 and 343)

$$
\mathbf{S}_{\mathrm{ac}}=p \mathbf{v},
$$

where $p$ is excess pressure and $\mathbf{v}$ is the field (or particle) velocity. The elastic field intensity $\mathbf{S}_{\mathrm{el}}$ is (Morse and Feshbach, 1953, pp. 151 and 345)

$$
\mathbf{S}_{\mathrm{el}}=-\frac{\partial \mathbf{s}}{d t} \cdot \mathfrak{T}
$$

where $\partial \mathbf{s} / d t$ is the particle velocity and $\mathcal{T}$ is the stress dyadic. In a homogeneous, isotropic, elastic medium there are two field intensities corresponding to compressional (longitudinal) and shear (transverse) waves, $\mathbf{S}_{\text {comp }}$ and $\mathbf{S}_{\text {shear }}$.

In a compressible, nonviscous fluid the field variable is velocity potential $(\psi)$, the field (or particle) velocity is $\nabla \psi$, and the pressure is $-\rho(\partial \psi / \partial t$ ) (Morse and Feshbach, 1953, p. 343). The velocity potential satisfies the scalar wave equation, which has a solution of the form $g(x-c t)$ for a plane wave propagating in the $x$ direction. The corresponding acoustic field intensity is

$$
\mathbf{S}_{\mathrm{ac}}=\mathbf{i} \rho c_{\mathrm{ac}}\left[g^{\prime}\right]^{2}=\mathbf{i}\left(p^{2} / \rho c_{\mathrm{ac}}\right)
$$

and the elastic field intensities [using solutions for the displacement and shear potentials of $f(x-c t)$ and $\mathbf{j} F(x-c t)$, respectively] are

$$
\begin{aligned}
& \mathbf{S}_{\text {comp }}=\mathbf{i}(\lambda+2 \mu) c_{c}\left[f^{\prime \prime}\right]^{2}=\mathbf{i}(\lambda+2 \mu) c_{c}[\boldsymbol{\nabla} \cdot \mathbf{u}]^{2}, \\
& \mathbf{S}_{\text {shear }}=\mathbf{i} \mu c_{s}\left[F^{\prime \prime}\right]^{2}=\mathbf{i} \mu c_{s}[\nabla \times \mathbf{u}]^{2} .
\end{aligned}
$$

Again, if the shear modulus vanishes, no shear waves are supported, $\lambda$ reduces to the bulk modulus, and the compressional wave speed reduces to the acoustic wave speed. In this case, $g^{\prime}\left(x-c_{c} t\right)=-c_{c} f^{\prime \prime}\left(x-c_{c} t\right)$ and the compressional field intensity reduces to the acoustic field intensity. In all cases, for isotropic media, the field intensity is a vector with a magnitude equal to the energy density $(w)$ times the wave speed $(c)$ and a direction normal to the wavefront (Morse 
and Feshbach, 1953, pp. 151 and 312). As defined here the field intensity varies with time as the wave passes a given point in the medium.

The expressions for energy density and field intensity were derived for plane waves in homogeneous media, but both properties are dependent only on the local material parameters and require only that the wave be planar in the vicinity of the measurement point. It is not unreasonable then to apply these expressions to propagation in helerogeneous media (Aki and Richards, 1980, p. 127).

Snapshots of the numerical scattering chamber display the compressional and shear "amplitude densities," $-\sqrt{\lambda+2 \mu} \boldsymbol{\nabla} \cdot \mathbf{u}$ and $-\sqrt{\mu} \boldsymbol{\nabla} \times \mathbf{u}$, respectively. For body waves in homogeneous or gradually changing material with nearly planar wavefronts, the magnitude of the amplitude density is the square root of the energy density, and the sign information in the waveform is retained. For interface waves the amplitude densities are still defined, but compressional and shear effects are coupled and the amplitude densities no longer have a simple relationship to energy. These are the same quantities that were plotted in the snapshots of Dougherty and Stephen (1988).

In the numerical scattering chamber, time series for receivers in the water column and in the elastic bottom record the normalized dilatation $(d),-(\lambda+2 \mu) \nabla \cdot u$, and the normalized rotation $(r),-\mu \nabla \times u$, not to be confused with the amplitude density defined above. In a fluid, where the shear modulus is zero, the normalized dilatation equals the pressure. Thus the normalized dilatation and rotation are the analogous quantities in a solid to the pressure in a fluid. The acoustic field intensity in the fluid and the compressional and shear field intensities in the solid are then simply

$$
\mathrm{S}_{\mathrm{ac}}=\mathbf{i} \frac{p^{2}}{\rho c_{\mathrm{ac}}}, \quad \mathrm{S}_{\mathrm{comp}}=\mathbf{i} \frac{d^{2}}{\rho c_{\mathrm{c}}}, \quad \mathrm{S}_{\text {shear }}=\mathbf{i} \frac{r^{2}}{\rho c_{s}} .
$$

Since field intensity is the basis for the determination of scattering coefficients, scattering out of the bottom of the numerical scattering chamber into the lower homogeneous solid can be treated in a fashion analogous to scattering up into the water column.

For many applications only the time average of the magnitude of the field intensity is of interest. It is assumed that the direction of propagation at a given point is constant over the time history of the wave and that locally the propagation can be treated as a plane wave in homogeneous media. Following Burdic (1991, p. 30), we refer simply to the intensity I:

$$
I-\langle|\mathbf{S}|\rangle=\langle w\rangle c .
$$

The intensity of a plane acoustic wave in a homogeneous medium with density $\rho$ and sound speed $c$ is (Burdic, 1991)

$$
I=p_{\mathrm{rms}}^{2} / \rho c,
$$

where $p_{\mathrm{rms}}$ is the root mean square of the pressure time series and it is uniform in space. For a spherical wave the intensity is

$$
I=\left(1 / r^{2}\right)\left(p_{0 . \mathrm{rms}}^{2} / \rho c\right),
$$

where $p_{0 . r m s}$ is the root-mean-square level of the pressure time series at unit distance from the source and $r$ is range from the source. At large ranges, the spherical wave can be considered locally planar and the two expressions for intensity are equivalent. The intensity level in decibels is

$$
L=10 \log _{10}\left(I / I_{\text {ref }}\right),
$$

where $I_{\text {ref }}$ is a reference intensity. For a pressure pulse in water, the reference intensity corresponds to a $p_{\mathrm{rms}}$ of $1 \mu \mathrm{bar}$ and, in cgs units, is $0.667 \times 10^{-12} \mathrm{~W} / \mathrm{cm}^{2}$. For wave propagation in heterogeneous media, we also assume that, over a vanishing small volume, the medium is homogeneous and the propagation is planar, so that the definition of intensity, (1), applies pointwise.

Aki, K., and Richards, P. G. (1980). Quantitative Seismology: Theory and Methods (Freeman, San Francisco), 1st ed.

Alford, R. M., Kelly. K. R., and Boore. D. M. (1974). "Accuracy of finitcdifference modeling of the acoustic wave equalion," Geuphysics 39, 834842.

Alierman, Z. S., and Karal, F. C., Jr. (1968). "Propagation of elastic waves in layered media by finite difference methods," Bull. Seismol. Soc. Am. 58, 367-398.

Berman, D. H. (1991). "Simulations of rough interface scattering," J. Acoust. Soc. Am. 89. 623-636.

Berman, D. H., and Perkins, J. S. (1990). "Rayleigh methods for scallering from random and deterministic inlerfaces," J. Acoust. Suc. Am. 88, 10321044.

Boore, D. M. (1970). "Finite difference solutions to the equations of elastic wave propagation with applications to Love waves over dipping interfaces," Ph.D. thesis, Massachusetts Institute of Technology-

Burdic, W. S. (1991). Underwater Acoustic System Analvsis (Prenlice-Hall, Englewood Cliffs, NJ), 2nd ed.

Burns, D. R., and Stephen, R. A. (1990). "Three-dimensional numerical modeling of geoacoustic scattering from seafloor topography," J. Accust. Soc. Am. 88, $2338-2345$.

Caruthers, J. W., and Bourgeois, B. (1992). "Geomorphic parameter estimations with neural networks using bathymetry and backscatter data," J. Acoust. Soc. Am. 91, 2428.

Cerjan, C., Kosloff, D., Kosloff, R., and Reshef, M. (1985). "A non reflecting boundary condition for discrete acoustic and elastic wave cquations," Geophysics 50, 7015-708.

Cervený, V., Popov, M. M.. and Pśenčik, I. (1982). "Computation of wave fields in inhomogeneous media-Gaussian beam approach," Geophys. J. R. Astron. Soc. 70, 109-128.

Chapman, C. H. (1978). "A new method for computing synthetic seismograms," Geophys. J. R. Astron. Soc. 54. 481-518.

Chapman, C. H. (1981). "Generalized radon transforms and slant stacks," Geophys. J. R. Astron. Soc. 66, 445-453.

Cheng. N. (1994). "3-D finite difference borehole wave propagation in isoIropic and anisotropic medium," Ph.D. thesis, Massachusetts Institute of Technology.

Chuang, S., and Johnson, R. K. (1982). "Acoustic wave scattering from a tluid/solid periodic surface," J. Acoust. Soc. Am. 71, $1368-1376$.

Clacrbout, J. F. (1970). "Coarse grid calculations of waves in inhomogeneous media with application to delineation of complicated seismic structure," Geophysics 35, 407-418.

Clayton, R., and Engquist, B. (1977). "Absorbing boundary conditions for acoustic and elastic wave equations," Bull. Scismol. Soc. Am. 67, 1529_ 1540.

Dablain, M. A. (1986). "The application of high-order differencing to the scalar wave equation," Geophysics 51, 54-66.

Dacol, D. K., and Berman, D. H. (1988). "Sound scattering from a randomly rough fluid-solid interface." J. Acoust. Soc. Am. 84, 292-302.

Day, S. M., and Minster, J. B. (1984). "Numerical simulation of atlenuated wavefields using a Padé approximant method," Geophys. J. R. Astron. Soc. 78, 105-118.

Dougherty, M. E., and Stephen, R. A. (1988). “Seismic energy partitioning and scattering in laterally heterogeneous occan crust," Pure Appl. Geophys. 128, 195-229. 
Dougherty, M. E., and Stephen, R. A. (1991). "Seismo/acoustic propagation through rough seafloors," J. Acoust. Soc. Am. 90, 2637-2651.

Durrani, T. S., and Bisset, D. (1984). "The Radon transform and its properties," Geophysics 49,1180-1187.

Emerman, S. H., and Stephen, R. A. (1983). "Comment on "Absorbing boundary conditions for acoustic and elastic wave equations,' by R. Clayton and B. Engquist," Bull. Seismol. Soc. Am. 73, 661-666.

Emmerich, H., and Korn, M. (1987). "Incorporation of atrenuation into time-domain computations of seismic wave tields," Geophysics 52, 1252 1264.

Engquist, B., and Majda, A. (1977). "Absorbing boundary conditions for the numerical simulation of waves," Math. Comp. 31, 629-651.

Fricke, J. R. (1991). “Acoustic scaltering from elastic ice: A finite difference solution," Ph.D. thesis, MIT/WHOI Joint Program in Oceanography

Fricke, J. R. (1993). "Acoustic scattering from elemental Arctic ice features: Numerical modeling results," J. Acoust. Soc. Am. 93, 1784-1796.

Gan, H., Levin, P. L., and Ludwig, R. (1993). "Finite element formulation of acoustic scattering phenomena with absorbing boundary condition in the frequency domain," J. Acoust. Soc. Am. 94. 1651-1662.

Gazdag, J. (1981). "Modeling of the acoustic wave equation with transform melhods," Geophysics 46, 854-859.

Gerstof.. P., and Schmide, H. (1991). "A boundary clement approach to ocean seismoacoustic facet reverberation," J. Acoust. Soc. Am. 89, 16291642.

Goff, J. A., and Jordan, T. H. (1988). "Stochastic modeling of seafloor morphology: Inversion of Sea Beam data for second-order stalistics," J. Geophys. Res. 93, 13 589-13 608.

Goff, J. A., and Jordan, T. H., (1990). "Pacific and Atlantic Models of Small-Scale Seafloor Topography (1)," Office of Naval Research Special Research Project on Acoustic Reverberation.

Haartsen, M. W., Bouchon, M., and Toksöz, M. N. (1994). "A study of seismic/acoustic wave propagation through a laterally varying multilayered medium using the boundary integral equation-discrete wavenumber method," to be published in J. Acoust. Soc. Am.

Higdon, R. L. (1986). "Absorbing boundary conditions for diffcrence approximations to the multidimensional wave equation," Math. Comp. 47, 437-459.

Higdon, R. L. (1991). "Absorbing boundary conditions for elastic waves," Geophysics 56, 231-241.

Jackson, D. R., and Briggs, K. B. (1992). "High-frequency bollom backscattering: roughness versus volume scattering," J. Acoust. Soc. Am. 92, 962-977.

Kappus, M. E., Harding, A. J., and Orcutt, J. A. (1990). “A comparison of tau-p transform methods," Geophysics 55, 1202-1215.

Kelly, K. R., and Marfurt, K. J. (eds.) (1990). Numerical Modeling of Seismic Wave Propagation (Society of Exploration Geophysicists, Tulsa, OK).

Kelly, K. R., Ward, R. W., Treitel, S., and Alford, R. M. (1976). "Synthetic seismograms: a finite-difference approach," Geophysics 41, 2-27.

Kennett, B. L. N. (1972). "Seismic wave scallering by obstacles on interfaces," Geophys. J. R. Astron. Soc. 28, 249-266.

Kosloff, D. D., and Baysal, E. (1982). "Forward modeling by a Fourier method," Geophysics 47, 1402-1412.

Kuperman, W. A., and Schmidt, H. (1986). "Rough surface elastic wave scattering in a horizontally stratified ocean," J. Acoust. Soc. Am. 79, $1767-1777$.

Kuperman, W. A., and Schmidt, H. (1989). "Self-consistent perturbation approach to rough surface scattering in stratified elastic media," J. Acoust. Soc. Am. 86, 1511-1522.

Lee, D., Botseas, G., and Papadakis, J. S. (1981). "Finite-difference solution of the parabolic wave equation," J. Acoust. Soc. Am. 70, 795-800.

Leslie, H. D., and Randall, C. J. (1992). "Multipole sources in boreholes penetrating anisotropic formations: numerical and experimental results." J. Acoust. Soc. Am. 91, 12-27.

Levander, A., Harding, A., and Orcutt. J. (1993). "Numerical scattering results for a rough, unsedimented seafloor," in Ocean Reverberation, edited by D. D. Ellis, J. R. Preston, and H. G. Urban (Kluwer, Dordrecht), pp. 215-220.

Levander, A. R. (1985). "Use of the telegraphy equation to improve absorbing boundary efficiency for fourth-order acoustic wave finile difference schemes," Bull. Seismol. Soc. Am. 75, 1847-1852.

Levander, A. R. (1988), "Fourth-order P-SV finite-difference seismograms," Geophysics 53, 1425-1436.

Lim, R., Lopes, J. L., Hackman, R. H., and Todoroff, D. G. (1993). "Scat- tering by objects buried in underwater sediments: theory and experiment," J. Acoust. Soc. Am. 93, 1762-1783.

Madariaga, R. (1976). "Dynamics of an expanding circular fault," Bull. Seismol. Soc. Am. 66, 639-666.

Matsumoto, H., Dziak, R. P., and Fox, C. G. (1993). "Estimation of seafloor microtopographic roughness through modeling of acoustic backscatter data recorded by multibeam sonar systems," J. Acousl. Soc. Am. 94 2776-2787.

Morse, P. M., and Feshbach, H. (1953). Methods of Theoretical Physics (McGraw-Hill, New York).

Murphy, J. E., and Chin-Bing. S. A. (1989). "A finite-element model for ocean acoustic propagation and scattering," J. Acoust. Soc. Am. 86, $1478-1483$

Ogilvy, J. A. (1987). "Wave scaltering from rough surfaces," Rep. Prog. Phys. 50, 1553-1608.

Orcutt, J., Harding, A., and Levander, A. (1993). "The effects of seafloor roughness on reverberation: finite difference and Kirchhoff simulations," in Ocean Reverberation, edited by D. D. Ellis, J. R. Preston, and H. G. Urban (Kluwer, Dordrecht). pp. 221-226.

Peng, C., and Toksoz, M. N. (1994). "An optimal absorbing boundary condition for finite difference modeling of acoustic and elastic wave propagation," J. Acoust. Soc. Am. 95, 733745.

Pierce, A. D. (1989). Acoustics: An Introduction to Its Physical Principles and Applications (Acoustical Society of America, Woodbury, NY).

Preston, J. R., Akal, T., and Berkson, J. (1990). "Analysis of backscallering data in the Tyrrhenian Sea," J. Acoust. Soc. Am. 87, 119-134.

Priolo, E., Carcione, J. M., and Seriani, G. (1994). "Numerical simulation of interface waves by high-order spectral modeling lechniques," J. Acoust. Soc. Am. 95, 681-693.

Schmidt, H., and Jensen, F. B. (1986). "Evaluation of experimental techniques for determining the plane wave reflection coefficient al the sea floor," in Ocean Seismo-acoustics: Low-Frequency Underwater Acoustics, edited by T. Akal and J. M. Berkson (Plenum, New York), pp. 721-730.

Stephen, R. A. (1983). "A comparison of finite difference and refleclivity seismograms for marine models," Geophys. J. R. Astron. Soc. 72, 39-57.

Stephen, R. A. (1988). "A review of finite difference methods for seismoacoustics problems at the seafloor," Rev. Geophys. 26, 445-458.

Stephen, R. A. (1990). "Solutions to range-dependent benchmark problems by the finite difference method," J. Acoust. Soc. Am. 87, 1527-1534.

Stephen, R. A. (1991). "A numerical scattering chamber for studying low angle seafloor backscatter," J. Acoust. Soc. Am. 90, 2276.

Stephen, R. A. (1993). "A numerical scattering chamber for sludying reverberation in the seafloor," in Ocean Reverberation, edited by D. D. Ellis, J. R. Preston, and H. G. Urban (Kluwer, Dordrecht), pp. 227-232.

Stephen, R. A., and Bolmer, S. T. (1985). "The direct wave root in marine seismology." Bull. Seismol. Soc. Am. 75, 57-67.

Stephen, R. A., and Dougherty, M. E. (1993). "Canonical seafloor models and the finite difference method for low-angle acoustic backscalter," in Computational Acoustics-Volume 1, edited by R. L. Lau, D. Lee, and A. R. Robinson (Elsevier, Amsterdam), pp. 227-246.

Stephen, R. A.. Pardo-Casas, F., and Cheng, C. H. (1985). "Finite-difference synthetic acoustic logs." Geophysics 50, 1588-1609.

Stephen, R. A., and Swift, S. A. (1994). "Finite difference modeling of geoacoustic interaction at anelastic seafloors," J. Acoust. Soc. Am. 95, $60-70$.

Stoffa, P. L., Buhl, P., Diebold, J. B., and Wenzel, F. (1981). “Direct mapping of seismic data to the domain of intercept time and ray parameter: a plane wave decomposition," Geophysics 46, 255-267.

Teng, Y.-C., and Kuo, J. T. (1988). "A finite element algorithm for solving the transient problem in a fluid-solid coupled medium," in Computational Acoustics: Wave Propagation, edited by D. Lee, R. L. Sternberg, and M. H. Schultz (Elsevier, Amsterdam), p. 422.

Thorsos, E. I. (1988). "The validily of the Kirchhoff approximation for rough surface scallering using a Gaussian roughness spectrum," J. Acoust. Soc. Am. 83, 78-92.

Thorsos, E. I., and Jackson, D. R. (1989). "The validity of the perturbation approximation for rough surface scattering using a Gaussian roughness spectrum," J. Acoust. Soc. Am. 86, 261-277.

Virieux, J. (1986). "P-SV wave propagation in helerogeneous media: velocity-stress finite-difference method," Geophysics 51, 889-901.

Yoon, K. H., and McMechan, G. A. (1992). "3-D finite-difference modeling of elastic waves in borehole environments," Geophysics 57, 793-804. 